\title{
Coherent Cherenkov radio pulses from hadronic showers up to EeV energies
}

\author{
Jaime Alvarez-Muñiz ${ }^{\mathrm{a}, *}$, Washington R. Carvalho Jr. ${ }^{\text {, }}$, Matías Tueros ${ }^{\mathrm{a}, \mathrm{b}}$, Enrique Zas ${ }^{\mathrm{a}}$ \\ ${ }^{a}$ Depto. de Física de Partículas E' Instituto Galego de Física de Altas Enerxías, Universidade de Santiago de Compostela, 15782 Santiago de Compostela, Spain \\ ${ }^{\mathrm{b}}$ Depto. de Física, Facultad de Ciencias Exactas, Universidad Nacional de La Plata, Argentina
}

\section{A R T I C L E I N F O}

\section{Article history:}

Received 12 May 2010

Received in revised form 3 October 2011

Accepted 4 October 2011

Available online 13 October 2011

\section{Keywords:}

High energy cosmic rays and neutrinos

High energy showers

Cherenkov radio emission

\begin{abstract}
A B S T R A C T
The Cherenkov radio pulse emitted by hadronic showers of energies in the EeV range in ice is calculated for the first time using full three dimensional simulations of both shower development and the coherent radio pulse emitted as the excess charge develops in the shower. A Monte Carlo, ZHAIRES, has been developed for this purpose combining the high energy hadronic interaction capabilities of AIRES, and the dense media propagation capabilities of TIERRAS, with the precise low energy tracking and specific algorithms developed to calculate the radio emission in ZHS. A thinning technique is implemented to allow the simulation of radio pulses induced by showers up to $10 \mathrm{EeV}$ in ice. The code is validated comparing the results for electromagnetic and hadronic showers to those obtained with GEANT4 and ZHS codes. The contribution to the pulse of other shower particles in addition to electrons and positrons, mainly protons, pions and muons, is found to be below $3 \%$ for $10 \mathrm{PeV}$ and above proton induced showers. The characteristics of hadronic showers and the corresponding Cherenkov frequency spectra are compared with those from purely electromagnetic showers. The dependence of the spectra on shower energy and high-energy hadronic model is addressed and parameterizations for the radio emission in hadronic showers in ice are given for practical applications.
\end{abstract}

(c) 2011 Elsevier B.V. All rights reserved.

\section{Introduction}

The observation of neutrinos of EeV-scale energies is one of the main priorities in Astroparticle Physics. The detection of neutrinos will open a new window to observe parts of the Universe shielded by large depths of matter, unaccessible to conventional astronomy. The measurement of diffuse neutrino fluxes will provide further clues to the identification of the sources of ultra-high energy cosmic ray production, their composition and their production mechanisms [1]. In addition such detections could have important implications for fundamental particle physics.

A very promising and cost-effective method to detect highenergy neutrino interactions was first proposed by Askar'yan in the 1960s [2]. The idea is to detect the Cherenkov radiation at radio wavelengths generated by the excess number of electrons in the cascade of particles resulting from a high-energy particle interaction in a dense medium transparent to radio waves. The development of this excess charge, due to the interactions with matter electrons, is often referred to as the Askar'yan effect. The effect has been experimentally confirmed in accelerator experiments at SLAC in media such as sand [3,4], rock salt [5] and ice [6], with results in good agreement with theoretical calculations [7]. At radio frequencies ( $\mathrm{MHz}-$ $\mathrm{GHz}$ ), the emission is coherent and the radiated power scales with the square of the primary particle energy. This makes this method

\footnotetext{
* Corresponding author.

E-mail address: jaime.alvarezmuniz@gmail.com (J. Alvarez-Muñiz).
}

very promising for the detection of neutrinos and cosmic rays of the highest energies (EeV) [8-10].

Several experiments have already exploited this technique searching for ultra-high energy neutrinos, but no positive detection has been reported so far. These include experiments using the ice cap at the South Pole, such as the ANITA balloon experiment [11-13] and the RICE array of antennas buried under the Antarctic ice $[14,15]$. Other arrays are beginning to be constructed, such as the Askar'yan Radio Array ARA [16] and ARIANNA [17]. There are also a number of projects using the Moon as target for neutrino interactions, along with radio telescopes on Earth as radiation detectors, such as the pioneering Parkes [18,19], GLUE [20], Kalyazin [21], NuMoon [22], LUNASKA [23] and RESUN [24].

The interpretation of data from these experiments requires a detailed knowledge of the magnitude, angular distribution and frequency-dependence of the emitted Cherenkov radiation, which can then be related back to the properties of the induced cascade. This calls for accurate simulations of the Fourier-spectrum of coherent Cherenkov radiation from EeV showers in different dense media. In the past, full simulations of electromagnetic (EM) showers in ice were done up to PeV energies, using the well-known and well-tested ZHS code $[9,25]$ and different versions of the GEANT code $[26,27]$. Also, full simulations of hadronic and neutrino-induced showers in ice were carried out up to $100 \mathrm{TeV}$ with GEANT [28]. Hybrid Monte Carlo [29] and thinning techniques [30] were also developed to simulate electromagnetic [31,32,34], hadronic $[33,34]$ and neutrino-induced showers $[34,35]$ above PeV energies 
(mainly in ice and water). Above these energies the LPM effect $[36,37]$ starts to be important in the media under experimental consideration $[37,38,31]$. Semi-analytical calculations of the radio-emission have also been performed [39,41]. Very recently, simulations of not only the Fourier-components of the spectrum but also of the radio-pulse in the time-domain emitted in electromagnetic showers, have been performed with the ZHS code[42].

Modeling hadronic showers up to EeV energies is of utmost importance for neutrino detection, since they are induced by all neutrino flavors in neutral current (NC) interactions, as well as in the hadronic vertex of charged current (CC) interactions of muon and tau neutrinos. Although at EeV energies only $20 \%$ of the neutrino energy is on average carried by the hadronic shower, these showers are known to be less affected by the LPM effect [33], and their Cherenkov emission does not suffer from the shrinking of the Cherenkov cone, which would otherwise reduce the solid angle for observation. On the other hand, mixed showers induced in CC electron neutrino interactions are composed of a purely electromagnetic shower, produced by an energetic electron carrying on average $\sim 80 \%$ of the energy of the neutrino, and a hadronic shower, initiated by the debris of the interacting nucleon. The advantage for neutrino detection of these types of interaction is that all the neutrino energy is channeled into the resulting shower. However, the observation of the electromagnetic shower is expected to be very difficult, since the LPM effect shrinks the angular distribution of the electric field, reducing dramatically the available solid angle for detection. For these reasons, experiments aiming at detecting neutrinos using the radio technique gain most of their acceptance from neutrino-induced hadronic showers [15,12,43].

Clearly, accurate simulations of hadronic showers with energies above which the LPM effect becomes effective in different dense media are needed. In this paper we present the first steps in that direction. We have used the well-known AIRES code [44] in combination with the TIERRAS package [45] to simulate proton-induced showers in dense media, such as ice. We have implemented the algorithms to calculate the Fourier components of the electric field produced by charged particle tracks in the shower, developed by Zas-Halzen-Stanev in the well-known and well-tested ZHS code $[9,25]$. The result is a flexible and powerful code named ZHAIRES, which allows the simulation of electromagnetic, hadronic and neutrino-induced showers in a variety of media, along with their associated coherent radio emission due to the Askar'yan effect.

It is important to stress that in $[31,33]$ the calculations of the emission properties of showers in ice up to EeV energies were only done in an approximate way. They were based on the Fouriertransform of simulated longitudinal and lateral shower profiles. With ZHAirES we can obtain the radio-pulse features in a consistent manner within a well-tested simulation, computing the electric field emitted by charged particles in the shower. In Ref. [28], GEANT simulations were used to calculate the radio-pulse properties in the same way as the ZHAireS code. However these calculations were limited to energies below $100 \mathrm{TeV}$ due to GEANT limitations, while with ZHAireS we can simulate showers at EeV energies and above. Also, and for the first time, the contribution to the radio pulse of charged pions, muons and protons is accounted for and quantified, and parameterizations of the frequency spectrum of the radio pulse due to Askar'yan effect in hadronic showers are presented. Previous parameterizations of hadronic showers exist in the literature [33], but these are based on less accurate simulations than those presented in this work.

This paper is structured as follows. In Section 2 we describe the new ZHAIRES code and compare its performance and results for electromagnetic showers with those of the well-tested codes GEANT 4 [46] and ZHS [25,32]. We also explore thinning techniques in the ZHAIrES code, essential for the simulation of $\mathrm{EeV}$ showers. Section 3 is devoted to the simulation of hadronic show- ers up to EeV energies, emphasizing their differences with respect to purely electromagnetic showers. Section 4 summarizes and concludes the paper. In Appendix A, we also give parameterizations of the intensity of the Fourier-spectrum of the Cherenkov electric field emitted in hadronic showers, as a function of shower energy and observation angle.

In this paper we concentrate on hadronic showers in ice. We defer to future papers the study of coherent radio emission from EM and neutrino-induced showers. However we show that our results for proton showers can be applied to approximately model the Cherenkov emission from hadronic showers induced in high-energy neutrino interactions. The code can also be applied to the simulation of radio emission in extensive air showers [50,51], despite the different emission mechanism $[48,49]$.

\section{Radio emission in electromagnetic showers using the ZHAIRES code}

Coherent Cherenkov emission from high-energy showers in a dense medium such as ice is due to the excess of electrons over positrons in the shower. This excess is mainly caused by the low energy scattering processes, such as Compton, Møller and Bhabha scattering, that incorporate electrons of the medium into the shower [25], as well as to positron annihilation. The characteristics of this emission are dependent on the lateral and longitudinal profiles of the shower[7]. The longitudinal profile affects mainly the emission at angles far from the Cherenkov angle. As the shape of this profile is governed by high energy interactions, radio emission is strongly affected by the LPM effect, which reduces the probability of high energy ( $E>1 \mathrm{PeV}$ in ice) EM interactions. The lateral profile affects the features of the spectrum close to the Cherenkov angle at frequencies typically above $\sim 1 \mathrm{GHz}$, and is mainly determined by low energy particle scattering.

Next we describe the key elements of the ZHAireS code, a flexible and powerful Monte Carlo that allows the simulation of electromagnetic, hadronic and neutrino-induced showers and their associated coherent Cherenkov radio emission due to the Askar'yan effect in a variety of media.

\subsection{The ZHAIRES code}

ZHAIRES is based on the well-known AIRES $^{1}$ code [44] which we have used in combination with the TIERRAS package [45] to simulate showers in dense media, such as ice. We have implemented in AIRES algorithms to calculate the Fourier components of the electric field produced by charged particle tracks in the shower. These algorithms are the same used in the well-known and well-tested ZHS code, developed by Zas et al. [9,25].

The standard procedure to simulate Cherenkov coherent radio emission due to the Askar'yan effect in an energetic shower is to divide all charged particle tracks in the shower, down to a relatively low threshold energy, in small steps which are approximated by straight lines in which particles travel at constant speed. A space-time position is associated with the end points of each step, along with the speed and the charge of the particle. This is all that is needed to calculate the electric field, and to properly take into account interference effects between all steps (for a detailed discussion and the relevant equations see $[25,47])$. The AIRES package [44] is a well established Monte Carlo extensive air shower simulation code, from which detailed information on the space-time positions of the tracked particles can be extracted. This allows the use of AIRES (v2.8.4a) to simulate the coherent radio emission due to any type of particle interaction. However, AIRES by itself can

\footnotetext{
AIRshower Extended Simulations
} 
only simulate showers in air, where the contribution of the Askar'yan mechanism to radio emission is thought to be small, and other processes, such as geo-synchrotron radiation, are expected to be more important [49]. In order to simulate showers in dense, dielectric media, we have used the TIERRAS package [45]. Originally designed to continue the simulation of an extensive air shower underground, TIERRAS also enables the simulation of particle cascades in other dense media, such as ice, sea water, rock and soil. TIERRAS accounts for the atomic and mass number dependence of the interaction cross-sections and energy-losses of baryons, mesons and leptons, and includes a detailed treatment of the LPM effect of special relevance for radio emission [38,31,33].

In this work we concentrate on showers developing in ice, with refraction index $n=1.78$ and density $\rho=0.924 \mathrm{~g} \mathrm{~cm}^{-3}$. Using the ZHAIRES + TIERRAS framework, we track $e^{ \pm}$with energies above $80 \mathrm{keV}$, and $\pi^{ \pm}, \mu^{ \pm}, p$ and $\bar{p}$ above $500 \mathrm{keV}$, neglecting radio emission for energies below these thresholds. ${ }^{2}$ These energy cuts are the lowest available in AIRES. We also do not account for the emission of other charged particles such as kaons, which as will be shown in Section 3 constitute a negligible contribution.

We used the minimum propagation step available in AIRES, which is typically much smaller than a radiation length. Directly from the AIRES routines we get the start and end points for each step, along with the charge of the particle and propagation time. From these tracks the radio emission is then calculated making extensive use of the formula for the frequency $(\omega)$ spectrum of the electric field $\vec{E}$, at a position $\vec{x}$ in the Fraunhofer limit of large observation distance $R$, derived in $[9,25]$ and reproduced here for self-consistency:

$\vec{E}(\omega, \vec{x})=\frac{e \mu_{r} i \omega}{2 \pi \epsilon_{0} c^{2}} \frac{e^{i k R}}{R} e^{i(\omega-\vec{k} \cdot \vec{v}) t_{1}} \vec{v}_{\perp}\left[\frac{e^{i(\omega-\vec{k} \cdot \vec{v}) \delta t}-1}{i(\omega-\vec{k} \cdot \vec{v})}\right]$,

where $\mu_{r}$ is the relative magnetic permeability of the medium, $\epsilon_{0}$ the free space permittivity, $c$ the speed of light in vacuum, $k=\omega n /$ $c$ is the wave vector in a medium with refractive index $n, \vec{v}$ is the particle velocity, $t_{1}$ the start time and $\delta t$ is the time interval between the end points of the track.

We recall that this equation has been obtained with the following convention for the Fourier transform of the electric field:

$\tilde{f}(\omega)=2 \int_{-\infty}^{\infty} f(t) e^{i \omega t} d t$

where the factor 2 corresponds to an unusual convention (this factor is usually either 1 or $\left.(2 \pi)^{-\frac{1}{2}}\right)$. This does not pose a problem as long as the inverse Fourier transform is done consistently (see [42] for more details).

The necessary division of the particle paths into piecewise linear tracks for radio pulse calculations can be performed in various ways, with shorter divisions allowing increasingly accurate parameterizations to higher frequencies at the expense of computer time [27]. In ZHAireS the splitting is finer than in the default ZHS [39], roughly 3-4 times more steps, which is accurate enough for our purposes up to $\sim 10 \mathrm{GHz}$ and even above, i.e. above the frequency at which the coherent behavior of the emitted signal is expected to break down in ice [7]. Shorter tracks will lead to a significant increase in computing time but at no improvement in accuracy.

Coherent Cherenkov radio emission in showers in dense media is mainly due to electrons and positrons with $\sim \mathrm{MeV}$ kinetic energies. For instance, $50 \%$ of the projected track length due to the excess of negative charge in the shower, a quantity known to be proportional to the coherent electric field, is produced by electrons with kinetic energy between $100 \mathrm{keV}$ and $\sim 6 \mathrm{MeV}$, with little contribution ex-

\footnotetext{
2 Note that due to the isotropically distributed directions of particles below these thresholds, their contribution to the electric field is expected to be very small [52].
}

pected below $100 \mathrm{keV}$ [25]. We have explicitely checked this in our simulations, and the results obtained agree with [25]. A very accurate treatment of this $\mathrm{keV}$ to $\mathrm{MeV}$ kinetic energy range is thus needed to obtain a precise determination of the radio emission intensity. However, in AIRES, electrons from knock-on (KO) electron and positron interactions, such as Bhabha and Møller scattering, are not tracked explicitly if the kinetic energy of the incident $e^{ \pm}$is below $K_{\text {low }}=1-2 \mathrm{MeV}$, and a continuous energy loss $d E / d X$ is used instead. This posed a difficulty, since the approximation was not accurate enough to describe the sub-MeV electrons, and also because in order to fully describe the low energy part of the shower electronic component, one should lower $K_{\text {low }}$ to explicitly simulate knock-on interactions for electrons down to $K_{\text {low }}=106 \mathrm{keV}$. These two issues made the parameterization of $d E / d X$ used in AIRES unsuitable for radio applications. To solve these problems, we calculate the continuous ionization loss explicitly for low energy electrons and positrons integrating the energy loss for secondaries of energy below $K_{\text {low }}=106 \mathrm{keV}$, as done in the ZHS code, and replace the $d E / d X$ parametrization implemented in AIRES with the actual Bethe-Heitler energy loss formula at low energies. We have checked that the $d E / d X$ obtained in this way is in very good agreement with that implemented in GEANT 4 [46], with maximum relative differences $\sim 1 \%$ when the same $K_{\text {low }}$ is used.

\subsection{Comparison to GEANT4 and ZHS}

In order to validate the ZHAIRES code we have performed several comparisons of its output in ice with that produced by the GEANT 4 [46] and ZHS [25] codes. We have concentrated our efforts in comparing the lateral (perpendicular to shower axis) development of the excess negative charge in the shower, as well as the tracklength due to this excess negative charge. For these comparisons, we raised the $e^{ \pm}$kinectic energy cuts to $106 \mathrm{keV}$, to match the thresholds used in GEANT4 and ZHS.

Two relevant quantities affecting significantly the longitudinal development of an electromagnetic shower are the pair production (PP) and bremsstrahlung (BR) cross sections. In order to compare the PP cross-sections in ZHS and ZHAIRES ${ }^{3}$, we obtained the photon mean free path (MFP) at several energies. The result is shown in the top panel of Fig. 1. Since photoproduction is not taken into account in ZHS, we disabled it temporarily in some ZHAIRES simulations in order to allow a direct comparison. Up to $1 \mathrm{PeV}$, the MFP for PP interactions obtained in ZHAIRES is very similar to that in ZHS, with differences $\sim 3 \%$, and is equal to (9/7) $X_{0} \sim 46 \mathrm{~g} \mathrm{~cm}^{-2}$, where $X_{0}=36.08 \mathrm{~g} \mathrm{~cm}^{-2}$ is the radiation length in ice. Above $\sim 1 \mathrm{PeV}$, the energy at which the LPM effect starts to produce a decrease in the PP cross section in ice, the average interaction depths begin to deviate from each other. For photon energies above $100 \mathrm{PeV}$, the ZHAIRES PP cross-section is around 30\% lower than the ZHS one, and the LPM effect turns on at a smaller energy in the ZHAireS (TIERRAS) code than in ZHS. We attribute this difference to details in the implementation of the LPM effect in both codes [53,25,37]. This will not be investigated further in this paper, since these differences are mostly irrelevant for the hadronic showers and radio pulses discussed below [33]. Above energies of order $1 \mathrm{EeV}$, the photoproduction cross-section becomes larger than the PP cross-section and the interaction length decreases with respect to the case in which only the LPM PP cross-section was accounted for. The energy at which this happens is however model-dependent $[55,56]$. The average interaction length when photoproduction interactions are enabled in ZHAIRES, and in CORSIKA are also shown for comparison.

The difference in the PP cross section is partly responsible for differences in some macroscopic shower observables, such as the posi-

\footnotetext{
${ }^{3}$ Note that ZHAIRES uses the same high energy cross-sections as the AIRES + TIER-
} RAS framework, including the treatment of the LPM effect. 

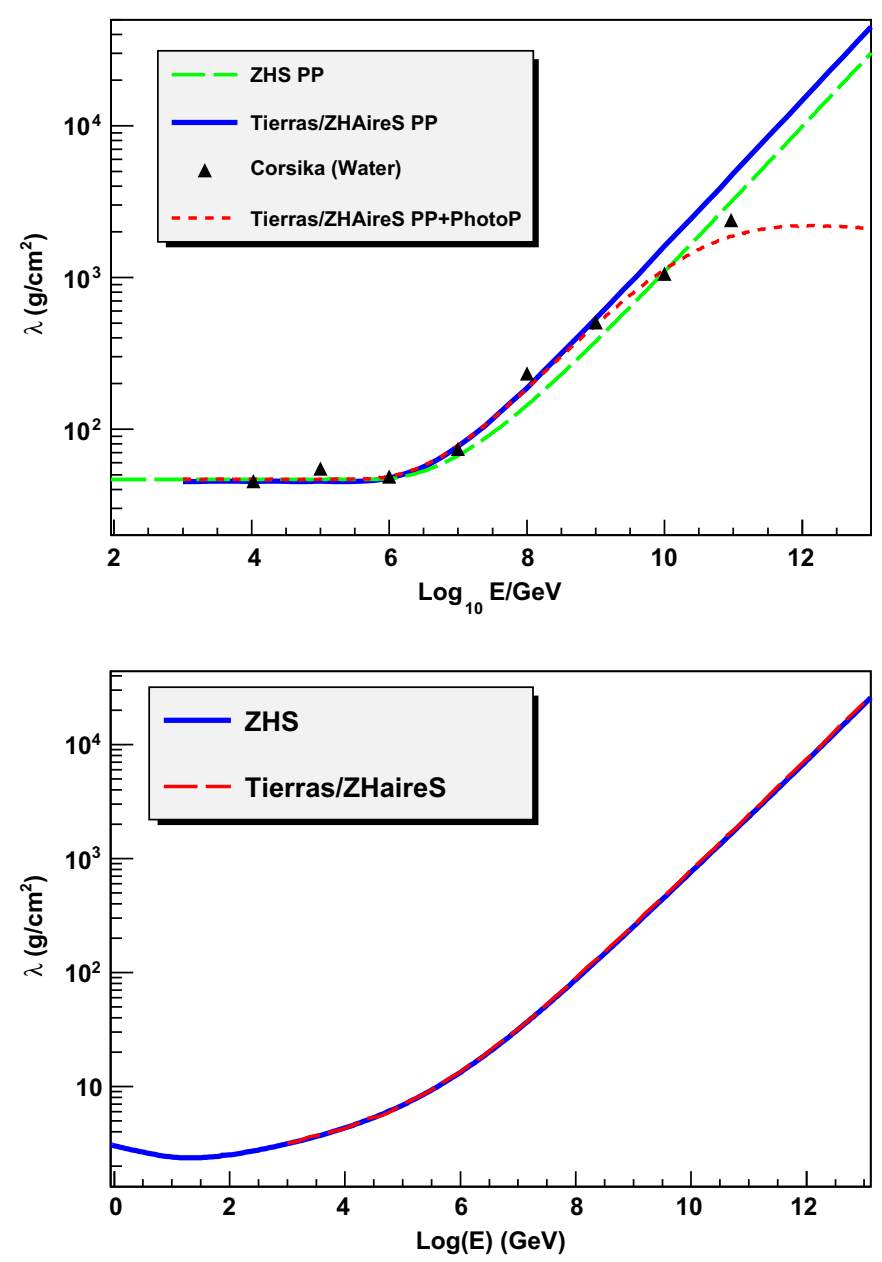

Fig. 1. Top panel: Mean free path $(\lambda)$ of photons at different energies in ZHS (long dashes) and ZHAIRES with (short dashes) and without (solid line) photoproduction interactions enabled. Also shown is the interaction length in CORSIKA [34] with both pair production and photoproduction enabled (triangles). Note that photoproduction is not accounted for in ZHS. Bottom panel: Mean free path of electrons of different energies in ZHS (blue solid line) and ZHAIRES (red dashed line overlapping the blue solid line). (For interpretation of the references to colour in this figure legend, the reader is referred to the web version of this article.)

tion $X_{\max }$ at which the number of particles in the shower is maximum. In Fig. 2 we show the average $X_{\max }$ in electron-initiated showers simulated with ZHS and ZHAIrES (with and without photoproduction interactions enabled). The PP cross-section at high energies is lower in ZHAIRES due to the difference in the strength of the LPM effect. This translates into an up to 20\% larger average $X_{\max }$ in ZHAIRES. If we disable the LPM effect and photoreactions in both ZHS and ZHAIRES, the elongation rate $\partial X_{\max } / \partial \log _{10} E$ becomes a constant, as expected, and we obtained a value of $81 \mathrm{~g} \mathrm{~cm}^{-2}$ in both cases.

In the bottom panel of Fig. 1 we also compare the mean free path for bremsstrahlung of electrons in ZHAIRES and ZHS simulations which agree to better than $\sim 5 \%$.

In Fig. 3 we show the lateral distribution of the excess negative charge, at a depth around shower maximum, obtained from simulations of $1 \mathrm{PeV}$ electron-induced showers in ice, performed using ZHAIRES and ZHS. The lateral distribution is a relevant quantity that mainly affects the frequency spectrum of the field at high frequencies, typically above $\sim 1 \mathrm{GHz}$. The agreement between both codes is rather $\operatorname{good}^{4}$.

\footnotetext{
${ }^{4}$ The differences near shower axis are mostly due to the fact that ZHS by default includes all particles with $r<0.1 \mathrm{~g} \mathrm{~cm}^{-2}$ in the first bin.
}

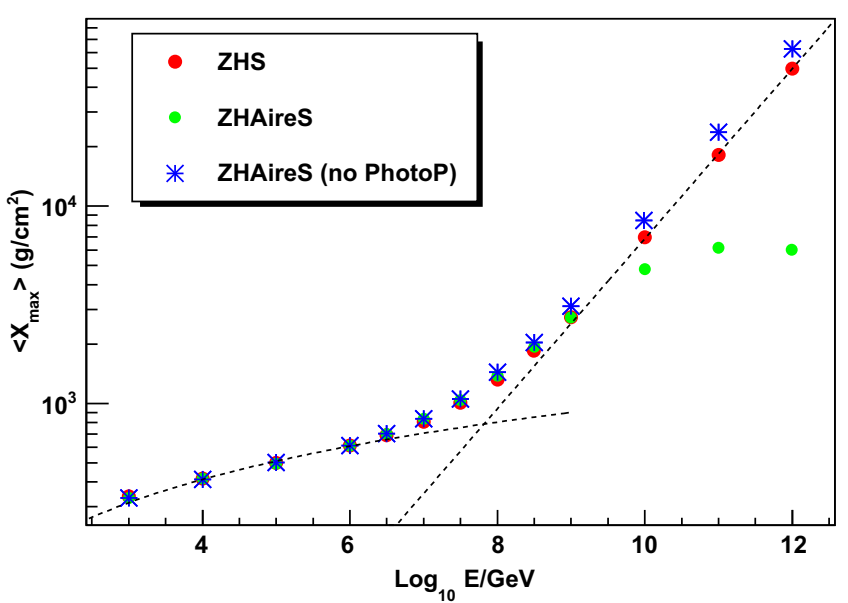

Fig. 2. Average depth of shower maximum $X_{\max }$ in electron-initiated showers simulated with ZHS (dark red dots) and ZHAIRES with (light green dots) and without (asterisks) photoproduction enabled. The dashed lines, shown just as a reference, are fits to ZHS data: a power law at high energy and a logarithmic lengthening (elongation rate) at lower energies. (For interpretation of the references to colour in this figure legend, the reader is referred to the web version of this article.)

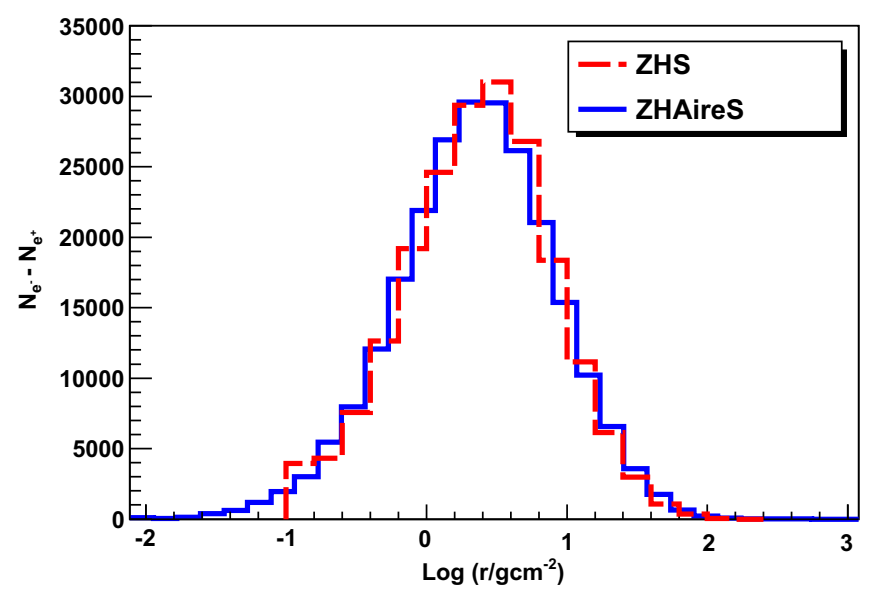

Fig. 3. Distribution of the excess negative charge as a function of the distance to the shower axis, obtained from simulations of $1 \mathrm{PeV}$ electron-induced showers in ice using ZHS (red dashed line) and ZHAIRE S (blue solid line). The lateral distribution refers to a depth around $X_{\max } \sim 600 \mathrm{~g} \mathrm{~cm}^{-2}$. See text for discussion. (For interpretation of the references to colour in this figure legend, the reader is referred to the web version of this article.)

In Table 1 we show the average track lengths obtained from 100 simulations of $100 \mathrm{GeV}$ electron-induced showers with ZHAIRES and ZHS, and compare them with GEANT results taken from [27]. The total track length is defined as the sum of all electron and positron tracks in the shower. The total projected track length is the sum of all the projections of these tracks onto the shower axis. The excess projected track length is the difference between the sum of all electron track projections minus the sum of all positron track projections. This is the most relevant quantity that determines the normalization of the electric field spectrum, since electrons and positrons contribute to the field with opposite signs. The relative differences between the total track length and excess projected track length as obtained in ZHAIRES and ZHS are $4.1 \%$ and $0.9 \%$, respectively. The corresponding differences between ZHAIRES and GEANT4 are $3.6 \%$ and $1.0 \%$, respectively ${ }^{5}$.

\footnotetext{
${ }^{5}$ Relative differences are calculated using [ZHAIRES-ZHS]/ZHS and [ZHAIRES GEANT4]/GEANT4.
} 
Table 1

Comparison between average track lengths obtained from simulations of electroninduced showers of $E_{0}=100 \mathrm{GeV}$ in ice, using ZHAIRES, GEANT 3.21, GEANT 4 and the latest version of ZHS [32]. The results of ZHAIRES and ZHS are the average of 100 simulated showers each, while the results of the GEANT 3.21 and GEANT 4 codes are taken from [27]. The total excess track length is $193.4 \mathrm{~m}$ in ZHAireS and $200.3 \mathrm{~m}$ in ZHS. Corresponding total excess track lengths for GEANT are not available. Note that the results shown here for the ZHS code give better agreement than reported in [27], due to a minor correction in the bremsstrahlung terms proportional to the density of matter electrons.

\begin{tabular}{lllll}
\hline MC code & ZHAireS & GEANT 3.21 & GEANT 4 & ZHS \\
\hline Total track $[\mathrm{m}]$ & 566.8 & 577.9 & 587.9 & 591.1 \\
Total projected $[\mathrm{m}]$ & 459.3 & 450.0 & 453.2 & 480.9 \\
Excess projected $[\mathrm{m}]$ & 123.9 & 123.5 & 122.7 & 125.1 \\
Excess projected/total & 0.219 & 0.214 & 0.209 & 0.212 \\
\hline
\end{tabular}

In Fig. 4 we compare the average frequency spectra of the Cherenkov radiation obtained from 20 ZHS simulations of electron-induced showers of primary energy $E_{0}=1 \mathrm{PeV}$, with the corresponding average spectrum obtained with the ZHAIRES code. One can see a very good agreement below the cut-off frequency in the spectrum (the frequency at which the electric field peaks before decreasing). Beyond the cut-off frequency the agreement worsens. At the Cherenkov angle and frequencies above about $3 \mathrm{GHz}$, the electric field is sensitive to the fine details of the shower, in particular to the different splitting of the charged particle tracks in ZHS and ZHAIRES codes. The finer splitting in the ZHA IRES code (3-4 times more steps) compared to ZHS [32] in which the electric field is calculated within the approximation "b" [39], leads to a higher spectrum in the $\mathrm{GHz}$ frequency range, when compared to ZHS. The effect of the splitting on the high-frequency Fourier components of the electric field was demonstrated numerically in [39]. At higher energies, typically above $1 \mathrm{EeV}$ or so photoproduction interactions start to play an important role in ice (see Fig. 2 and Ref. [40]). While in the ZHS code only EM processes are accounted for we expect the results of the ZHAireS and ZHS code for radio emission in EM showers to differ. This topic will be studied in a forthcoming paper [52].

\subsection{Thinning in ZHAIRES}

In order to make possible the simulation of showers above PeV energies, AIRES uses thinning algorithms $[30,44]$ to reduce computing time significantly. The basic idea of thinning is to follow only a small, representative fraction of the particles in a shower, and assign to each tracked particle a corresponding weight to compensate for the rejected ones [30].

Based on the properties of radio emission, a study was made in [32] of a thinning technique for calculations of radio emission in EM showers. The conclusion was that applying a thinning algorithm similar to the original Hillas algorithm [30], but with two thinning thresholds instead of one, i.e., thinning only particles with energies between $E_{\min }$ and $E_{\max }$, produces an accurate representation of the coherent Cherenkov radio-emission in electromagnetic showers, while at the same time keeps computing time to an acceptable value. The thinning parameters obtained in [32] were $E_{\min } \sim 100 \mathrm{MeV}-1 \mathrm{GeV}$ and $E_{\max } \sim\left(10^{-4}-10^{-5}\right) E_{0}$, where $E_{0}$ is shower energy. These parameters were obtained as a compromise between accuracy (smaller than $\sim 10 \%$ ) and CPU time. This algorithm is now implemented in the ZHS code.

The ZHAIRES code uses the same thinning algorithm as AIRES, with an energy threshold $E_{\mathrm{th}}$, equivalent to $E_{\max }$ in ZHS, and a socalled weight factor $W_{f}$ [44], which sets a maximum weight allowed in the simulation. It is important to remark that the weight factor is not the maximum weight of the particles in the simulation, and that the maximum weight increases linearly with shower

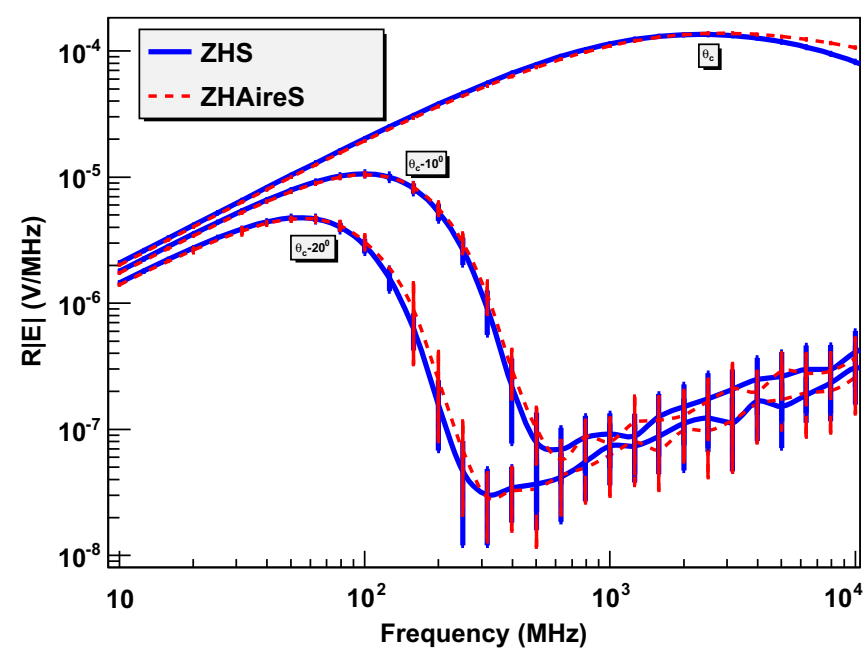

Fig. 4. Average frequency spectrum of the Cherenkov radiation obtained in simulations of 20 electron-induced showers with primary energy $E_{0}=1 \mathrm{PeV}$, using ZHS (blue solid line) and ZHAIRES (red dashed line). The spectrum is shown at three observation angles with respect to the shower axis. The RMS of the 20 simulated showers is also shown. (For interpretation of the references to colour in this figure legend, the reader is referred to the web version of this article.)

energy [44]. By fine-tuning $E_{\mathrm{th}}$ and $W_{f}$, one can make $W_{f}$ work in a similar fashion as $E_{\min }$, thus making the ZHAIRES thinning algorithm work in a similar way as the one in ZHS.

The key point is to select thinning parameters $\left(E_{\mathrm{th}}\right.$ and $W_{f}$, in the case of the ZHAIrES code), so that the computing time is minimized, while maximizing the agreement between full simulations, i.e., those performed following all particles, and the thinned ones. For that purpose we have compared full simulations of 20 showers at $1 \mathrm{PeV}$ with thinned simulations using different choices of $E_{\mathrm{th}}$ and $W_{f}$, in a similar fashion to what was done in [32]. A good compromise between accuracy and computing time was obtained for the parameters $E_{\mathrm{th}}=10^{-4} E_{0}$ (as in the case of ZHS [32]) and $W_{f}=0.06$. An example is shown in Fig. 5 , where we plot a comparison between 20 thinned simulations of electron-induced showers

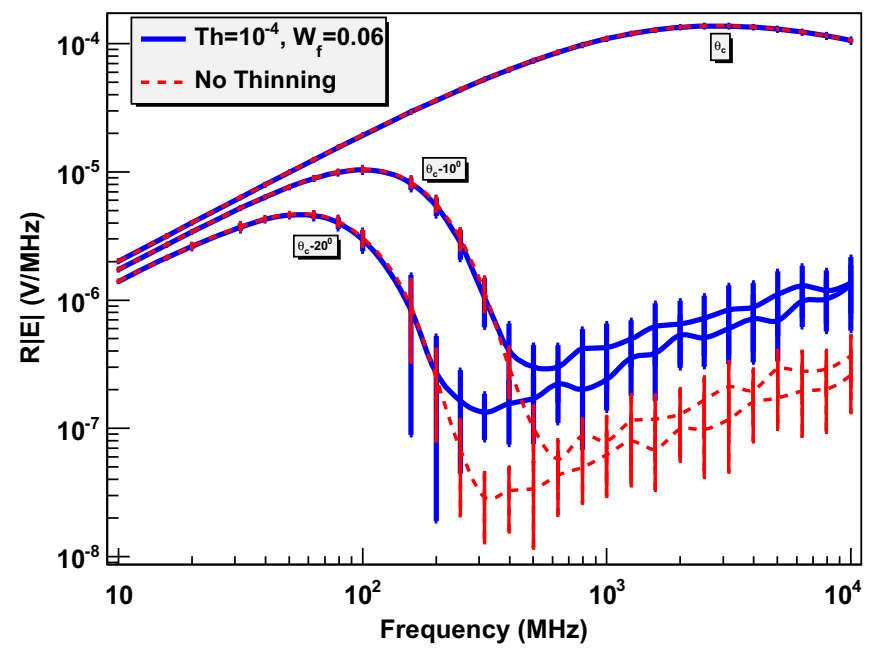

Fig. 5. Average frequency spectrum of the Cherenkov radiation generated by electron-induced showers with primary energy $E_{0}=1 \mathrm{PeV}$, obtained from 20 unthinned simulations (red dashed line) and 20 thinned simulations (blue solid line), using the ZHAIRES code. The spectrum is shown at three observation angles with respect to shower axis. The RMS of the 20 simulated showers is also shown. The parameters used in the thinned simulation were $E_{\mathrm{th}}=10^{-4} E_{0}$ and $W_{f}=0.06$. (For interpretation of the references to colour in this figure legend, the reader is referred to the web version of this article.) 
of energy $1 \mathrm{PeV}$ performed with the thinning parameters above, and 20 full simulations of the same energy, both using the ZHAIRES code. One can see that the agreement is very good up to frequencies beyond the cutoff frequency in the spectra, which depends on the observation angle. In this region, the difference between the average electric field in thinned and full simulations is always below $4 \%$, and the difference in the RMS of the simulated showers normalized to the average value, i.e., $\left(\sigma_{E}^{\text {thin }}-\sigma_{E}^{\text {unthin }}\right) /\langle|\vec{E}|\rangle$ with $\langle|\vec{E}|\rangle$ the electric field, is always smaller than $\sim 5 \%$. However, for observation angles away from the Cherenkov angle and frequencies well beyond the cutoff in the spectrum, the two calculations deviate significantly. This is not surprising since the Fourier components are sensitive to the fine structure of the shower (even at the individual particle level) in this angular and frequency region, and clearly the thinning algorithm in the ZHAIRES code is unable to account for it. This is not a problem because in that region the electric field strength is typically a factor 10 to 100 smaller than in the coherent region, and it is not expected to contribute significantly to the total emitted power.

At energies above $\sim 1-10 \mathrm{PeV}$, simulating showers without thinning becomes impractical, since computing time scales linearly with shower energy. This makes a direct comparison between thinned and un-thinned simulations above 1-10 PeV almost impossible. Instead, we have compared ZHAIRES thinned simulations, using the same parameters described before, with thinned simulations performed with ZHS, using its own thinning parameters $\left(E_{\min }\right.$ and $\left.E_{\max }\right)$, for which a comprehensive study of their validity was made [32]. The agreement between the two simulations is excellent at all energies, and we adopt the thinning parameters $\left(E_{\mathrm{th}}=10^{-4} E_{0}, W_{f}=0.06\right)$ in our subsequent simulations.

\section{Radio emission in hadronic showers}

In this section we study the coherent Cherenkov radio emission of hadronic showers in ice. Using the ZHAIRES code, we simulated large samples of proton-induced showers up to an energy of $E_{0}=10 \mathrm{EeV}$.

A first important check to perform is whether the values for the thinning parameters $E_{\text {th }}$ and $W_{f}$, obtained using EM showers in Section 2.3, are also valid to accurately calculate radio pulses from hadronic showers. For this purpose, in Fig. 6 we compare 20 full simulations of proton-induced showers with 20 showers simulated using the same thinning parameters obtained for electromagnetic showers. The agreement between both simulations is equivalently

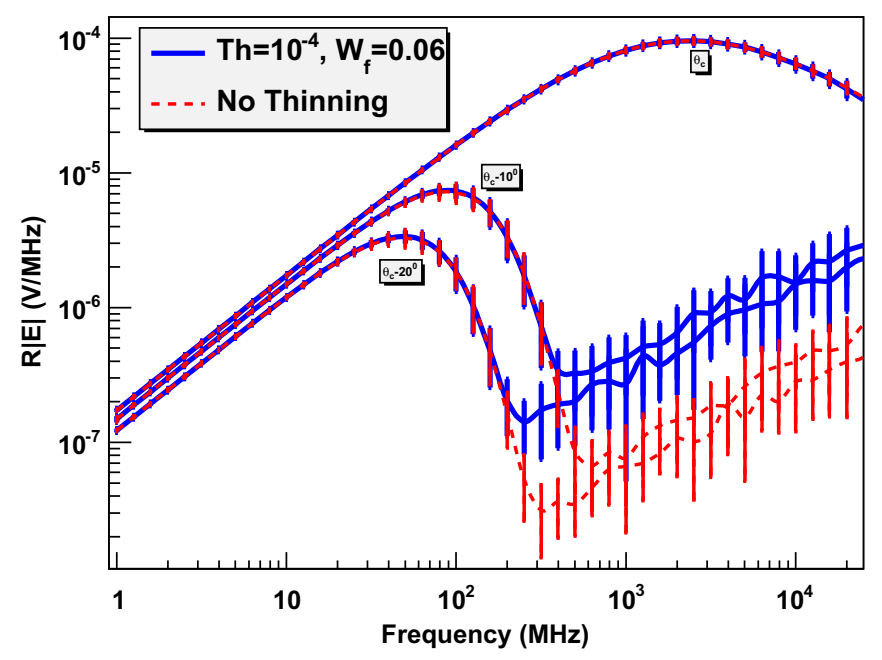

Fig. 6. Same as Fig. 5 for proton-induced showers. good except again for the frequency region above the region of full coherence. The CPU time required remains within reasonable values, reinforcing the conclusion that the same thinning algorithms and parameters can be used safely and provide a good description of both, electromagnetic and hadronic showers.

\subsection{Comparison to GEANT4 simulations}

In this section we compare our results to simulations of protoninduced showers using GEANT4, as reported in [28]. In Fig. 7, we compare the projected excess track length obtained in simulations of proton-induced showers using GEANT4 and ZHAIRES. For clarity, the projected excess track length has been divided by shower energy $E_{0}$. For the purposes of the discussion below, we also show in Fig. 7 the excess projected track length in the case of electron-induced showers, obtained in ZHAIRES.

GEANT4 simulations of proton showers have only been performed up to $90 \mathrm{TeV}$, while we performed ZHAIRES simulations up to $10 \mathrm{EeV}$, an energy 5 orders of magnitude higher. One can see that there is a good agreement between the results of both simulations. The small differences between the track lengths from both simulations, up to a maximum of $\sim 7 \%$, are well within 1 RMS of the ZHAIRES results, which were obtained from 20 simulations for each primary energy. In the case of the GEANT4 simulations reported in [28], a RMS < 10\% is quoted, except for $90 \mathrm{TeV}$, for which only a single shower was simulated and thus the RMS could not be obtained.

A feature that can be seen in Fig. 7 is that the track length due to the excess of electrons in hadronic showers approaches that in electron-induced showers at high energies. This is due to the fact that the fraction of primary energy going into the electromagnetic component of the shower increases with shower energy. Assuming energy equipartition between pions, in each interaction a fraction of $1 / 3$ of the incident pion energy would go into the electromagnetic component. Because the medium is dense, the produced charged pions are expected to interact before decaying and hence produce more $\pi^{0} \mathrm{~s}$ that contribute further to the EM content of the shower. As shower energy increases more particle generations and interactions occur, and hence more energy is transferred to the EM component. Hadronic showers at the highest energies will have a markedly electromagnetic character because of the high energy

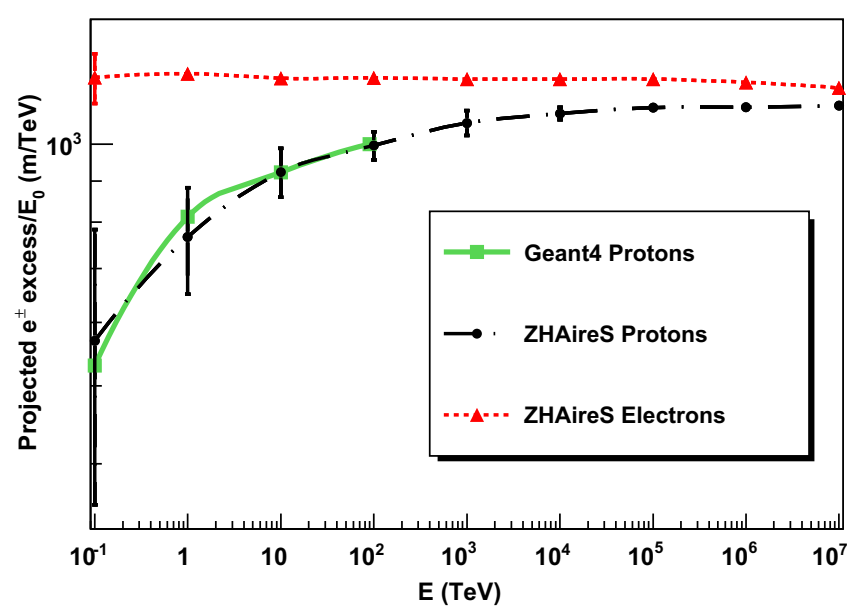

Fig. 7. Projected excess electron track length as a function of energy, obtained in GEANT4 [28] (solid line) and ZHAIRES (dot-dashed line) proton-induced shower simulations. For clarity, the track length has been divided by shower energy. Also shown are the track length for electron-induced showers, simulated using ZHAIRES. The GEANT4 results have an uncertainty (not plotted) of $\sim 10 \%$ [28]. 
involved and the medium density which prevents decays of most of the charged pions.

To further check this interpretation we have explicitly obtained in ZHAIRES simulations the energy going into the EM component of the shower. The result of this study shows that the increase with shower energy of the EM energy fraction is responsible for the increase with energy of the excess electron track in proton-induced showers shown in Fig. 7. Between 1 and $90 \mathrm{TeV}$, the ratio between the GEANT4 $e^{ \pm}$track length calculation, as shown in Fig. 7, and the EM energy fraction obtained from ZHAIRES simulations varies only slightly, between 11.6 and $11.8 \mathrm{~m} / \mathrm{TeV}$, showing that the projected $e^{ \pm}$excess track length is proportional to the EM energy fraction of the shower. We have also checked that the total track length behaves similarly, i.e. it is proportional to the EM energy fraction, confirming that the behavior seen in Fig. 7 is not an effect of projecting the track along the shower axis. The EM energy fractions obtained here are also in agreement with those obtained in [33], where a simplified simulation of shower development was performed.

Interestingly, in the case of electron-induced showers, one can see in Fig. 7 a small deviation from the linear dependence of the track length with energy. We attribute this behavior to photonuclear interactions becoming relevant at high energy and contributing to the hadronic component (i.e. reducing the EM energy fraction), even in electron-induced showers. This is confirmed by our simulations, as can be seen by the values of the EM energy fraction in electron induced showers, shown in Table 2.

\subsection{Radio emission}

The dominant radiation mechanism in hadronic showers is the same as in electron-induced ones, namely, emission of coherent Cherenkov radiation from the excess of electrons over positrons, i.e., the Askar'yan effect. However, there are differences between hadronic and electromagnetic showers in the normalization of the magnitude of the electric field, as well as in some important features of the frequency spectrum, which stem mainly from the different spatial distribution and from the fraction of EM energy in both types of showers [33,28].

Fig. 7 displays the projected track length excess divided by primary shower energy as a function of shower energy for both electromagnetic and hadronic showers. This ratio is almost constant for electromagnetic showers while for hadronic showers it

Table 2

Top table: Average total track lengths (top left) and projected track lengths (top right) divided by $E_{0}$ for different particle species obtained from simulations of protoninduced showers of $E_{0}=1 \mathrm{TeV}, 1 \mathrm{PeV}$ and $1 \mathrm{EeV}$ in ice, using ZHAIrES. Also shown in parenthesis are the RMS values for the 20 showers. Bottom table: Fraction of shower energy feeding the EM component for proton- and electron- induced showers of $E_{0}=1 \mathrm{TeV}, 1 \mathrm{PeV}$ and $1 \mathrm{EeV}$. The cuts used in these simulations were $80 \mathrm{keV}$ for $e^{ \pm}$and $500 \mathrm{keV}$ for hadrons.

\begin{tabular}{|c|c|c|c|c|c|c|c|}
\hline & \multicolumn{4}{|c|}{ Total track/ $E_{0}[\mathrm{~m} / \mathrm{TeV}]$} & \multicolumn{3}{|c|}{ Proj. track/E $E_{0}[\mathrm{~m} / \mathrm{TeV}]$} \\
\hline & \multicolumn{2}{|c|}{$1 \mathrm{TeV}$} & $1 \mathrm{PeV}$ & $1 \mathrm{EeV}$ & $1 \mathrm{TeV}$ & $1 \mathrm{PeV}$ & $1 \mathrm{EeV}$ \\
\hline$e^{+}$ & \multicolumn{2}{|c|}{$1271(145)$} & $1679(43)$ & $1747(16)$ & $1024(158)$ & $1466(47)$ & $1539(17)$ \\
\hline$e^{-}$ & \multicolumn{2}{|c|}{$2629(287)$} & $3440(86)$ & $3573(32)$ & $1801(262)$ & $2544(79)$ & $2664(30)$ \\
\hline$\pi^{+}$ & \multicolumn{2}{|c|}{$280(69)$} & $93(20)$ & $62(7)$ & $195(50)$ & $65(14)$ & $43.1(5.2)$ \\
\hline$\pi^{-}$ & \multicolumn{2}{|c|}{$277(74)$} & $90(20)$ & $60(7)$ & $184(51)$ & $59(13)$ & $39.6(4.8)$ \\
\hline$\mu^{+}$ & \multicolumn{2}{|c|}{$14.7(7.3)$} & $5.1(1.2)$ & $3.6(0.6)$ & $11.0(6.8)$ & $3.8(0.9)$ & $2.4(0.4)$ \\
\hline$\mu^{-}$ & \multicolumn{2}{|c|}{$14.4(7.2)$} & $5.0(1.5)$ & $3.3(0.4)$ & $9.6(6.1)$ & $3.6(1.3)$ & $2.16(0.35)$ \\
\hline$p$ & \multicolumn{2}{|c|}{$85(24)$} & $27.9(6.3)$ & $18.6(2.2)$ & $68(20)$ & $21.6(4.9)$ & $14.4(1.7)$ \\
\hline \multirow[t]{2}{*}{$\bar{p}$} & & & $0.3(0.2)$ & $0.19(0.03)$ & $0.3(0.9)$ & $0.3(0.2)$ & $0.18(0.03)$ \\
\hline & & \multicolumn{3}{|c|}{$E M^{\text {fraction }}$ proton-induced (\%) } & \multicolumn{3}{|c|}{$E M^{\text {fraction }}$ electron-induced (\%) } \\
\hline \multicolumn{2}{|c|}{$1 \mathrm{TeV}$} & \multicolumn{3}{|l|}{68.7} & \multicolumn{2}{|l|}{99.6} & \\
\hline \multicolumn{2}{|c|}{$1 \mathrm{PeV}$} & \multicolumn{3}{|l|}{89.2} & \multicolumn{2}{|l|}{99.2} & \\
\hline \multicolumn{2}{|c|}{$1 \mathrm{EeV}$} & \multicolumn{3}{|l|}{92.6} & \multicolumn{2}{|l|}{98.0} & \\
\hline
\end{tabular}

increases with energy because the EM energy ratio of hadronic showers also increases with energy, as explained before, approaching the value for electromagnetic showers but remaining always smaller. This reflects itself in the normalization of the electric field which is smaller in hadronic showers. This can be appreciated in the top panel of Fig. 8, where the Cherenkov spectrum of proton and electromagnetic $1 \mathrm{PeV}$ showers are compared. At this energy,
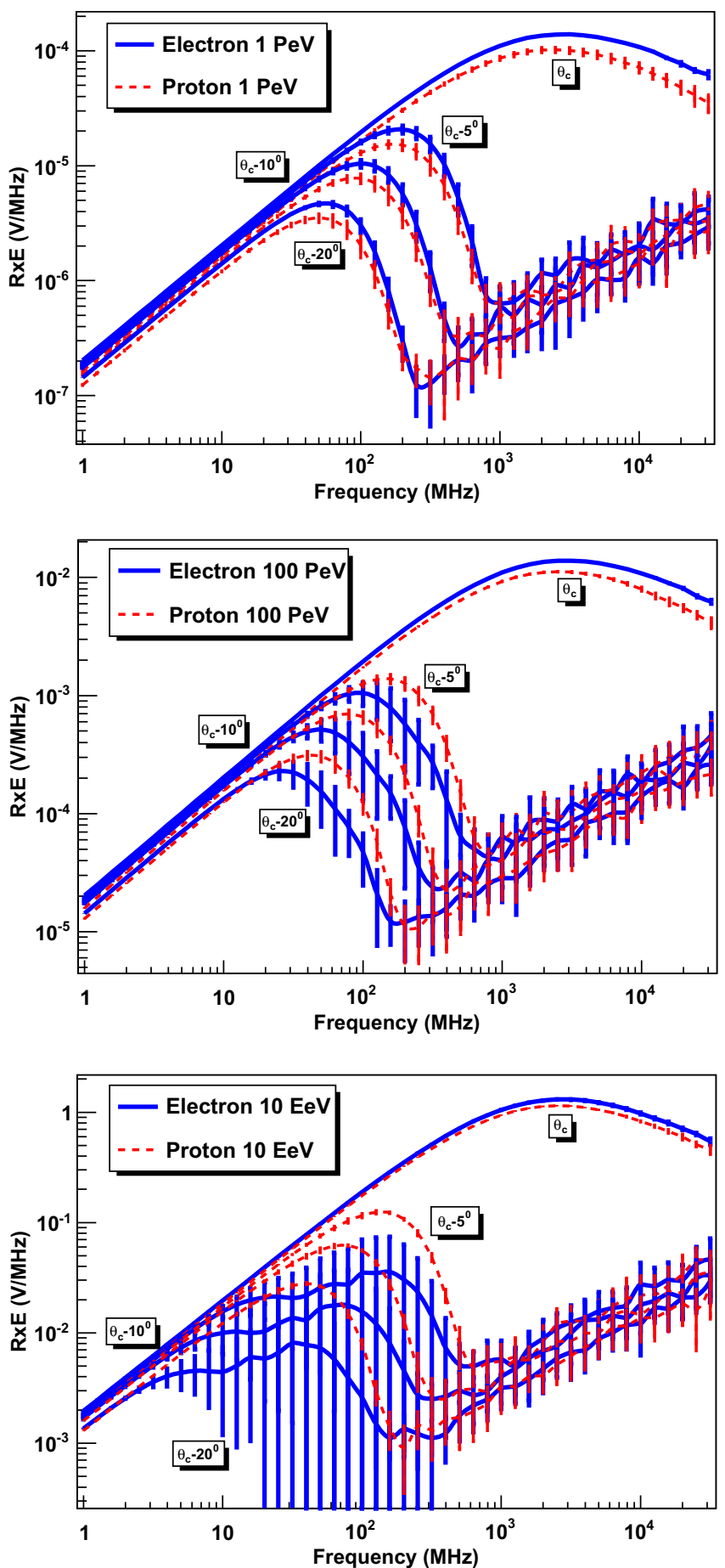

Fig. 8. Average frequency spectrum of the Cherenkov radiation obtained in ZHAIRES simulations of electron (solid blue line) and proton (dashed red line) showers with primary energy $E_{0}=1 \mathrm{PeV}$ (top panel), $E_{0}=100 \mathrm{PeV}$ (middle panel) and $E_{0}=10 \mathrm{EeV}$ (bottom panel). The spectrum is shown at four observation angles with respect to the shower axis. The RMS of the 20 simulated showers is also shown. (For interpretation of the references to colour in this figure legend, the reader is referred to the web version of this article.) 
the ratio between the electric fields at $1 \mathrm{MHz}$ and at the Cherenkov angle of the proton and electron induced showers is 0.864 , whilst the ratio between the EM energy fraction of these showers is very similar, 0.902 , i.e. $\left\langle|\vec{E}|_{p}\right\rangle /\left\langle|\vec{E}|_{e}\right\rangle \simeq E M_{p}^{\text {fraction }} / E M_{e}^{\text {fraction }}$. It can be also noted that as the shower energy increases (from top to bottom panels), the electric field produced by proton-induced showers approaches that of electromagnetic showers at small frequencies, where the coherence is full (10-100 MHz depending on the observation angle), as expected from the results of the excess projected track length displayed in Fig. 7.

A relevant feature of the frequency spectrum is the cut-off frequency at the Cherenkov angle, which is known to be inversely proportional to the lateral dimensions of the shower [7]. Hadronic showers typically spread over a larger lateral distance than electromagnetic ones [28], and as a consequence, the cut-off frequency in the hadronic case is expected to be smaller. The Cherenkov spectrum of electron-induced showers peaks at $\sim 3 \mathrm{GHz}$ for observation at the Cherenkov angle, while the peak shifts to lower frequencies, around $2.5 \mathrm{GHz}$, in proton-induced showers. The effect is however difficult to appreciate in Fig. 8.

Another important effect is the shift in the cut-off frequency at angles away from the Cherenkov angle, which is known to be determined by the longitudinal development of the shower $[7,25]$. Electron-induced showers develop more slowly than hadronic ones and hence penetrate more in the medium. As a result, for a fixed energy, hadronic showers are on average shorter in the longitudinal direction (along the shower axis) and we expect the cut-off frequency of their spectra, away from the Cherenkov angle, to be typically larger than in electron-induced showers. This effect is however not visible at energies below the LPM scale ( $\sim \mathrm{PeV})$ (top panel of Fig. 8), but it is apparent at energies above which the LPM effect becomes important for shower development.

Hadronic showers are known to be less affected by the LPM effect than electromagnetic ones [33], the reason is that the production of high energy photons through neutral pion decays is suppressed above PeV energies, since these energetic pions are more likely to interact than to decay. As a result, while electromagnetic showers are dramatically stretched in the longitudinal direction, hadronic showers grow much more slowly with energy. The immediate consequence is that, for a fixed observation angle away from the Cherenkov cone, the cut-off frequency is significantly smaller in electromagnetic than in hadronic showers. This is apparent in Fig. 8: At $100 \mathrm{PeV}$ and $10^{\circ}$ inside the Cherenkov cone, the cut-off frequency is $\sim 50 \mathrm{MHz}$ in the electron-induced showers, while it is $\sim 80 \mathrm{MHz}$ in the proton-induced ones. When EeV energies are reached, this effect becomes very strong. Moreover, shower-to-shower fluctuations of the longitudinal spread become very large due to the LPM effect in electron-induced showers [37]. As a result, some individual electromagnetic showers have a cut-off frequency even 10 times smaller than those in hadronic showers. In Fig. 8, the vertical bars represent the RMS of the average of 20 showers, making apparent that the RMS is much larger in electromagnetic than in hadronic showers for angles away from the Cherenkov one, for essentially all frequencies. It can also be appreciated that fluctuations significantly increase as shower energy increases in the case of electron-induced showers, while in hadronic showers the dependence on energy is weak.

We compare hadronic showers among themselves in Fig. 9, where we have divided the electric field by shower energy to make the differences between the features of the spectrum more apparent at different energies. The cut-off frequency at the Cherenkov angle increases slowly as the shower energy rises, since it is determined by the overall lateral spread of the shower, which becomes slightly narrower as the energy increases. Away from the Cherenkov angle, one can clearly see that the cut-off frequency decreases with energy as expected, but not so rapidly as in electromagnetic

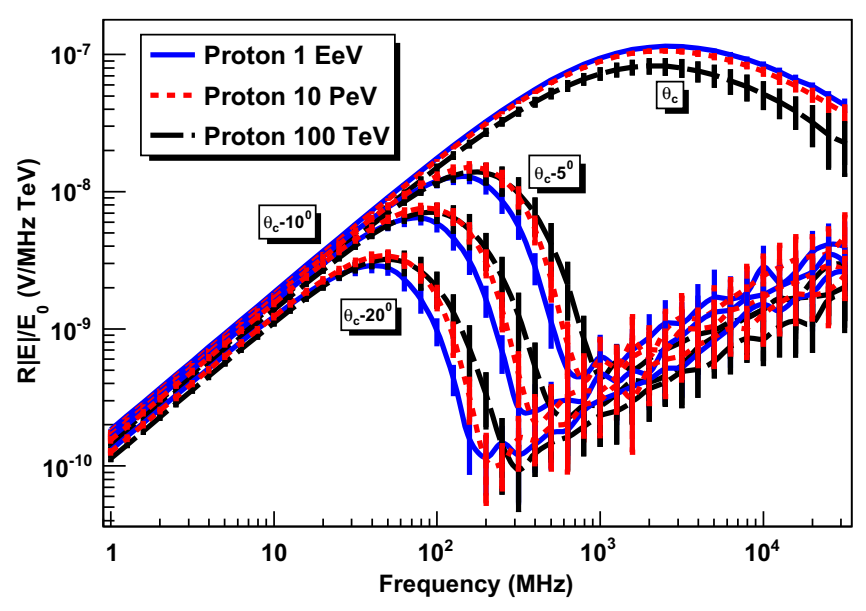

Fig. 9. Same as Fig. 8, but showing only proton-induced showers at energies $E_{0}=100 \mathrm{TeV}$ (long-dashed black line), $10 \mathrm{PeV}$ (dashed red line) and $1 \mathrm{EeV}$ (solid blue line). The electric field was divided by shower energy. (For interpretation of the references to colour in this figure legend, the reader is referred to the web version of this article.)

showers, because in hadronic showers the longitudinal spread increases only logarithmically with energy.

In Appendix A we give parameterizations of the frequency spectrum of the Cherenkov electric field emitted in hadronic showers, for practical applications, and we discuss their range of validity. The magnitude of the electric field is given as a function of proton energy, observation angle and frequency. As an example we show in Fig. 10 the electric field spectrum at the Cherenkov angle in a $100 \mathrm{PeV}$ proton shower, compared to the corresponding parameterization as given in Appendix A. As stated in Appendix A, one can see that the parameterizations work very well (accuracy $\sim 1 \%$ ) for frequencies up to the frequency $v_{\max }$, at which the spectrum is maximum for each observation angle. For frequencies above $v_{\text {max }}$, the accuracy worsens gradually and reaches $\sim 5 \%$ at $v=2 v_{\max }$, for observation at the Cherenkov angle $\theta=\theta_{C}$, and $\sim 15 \%$ at angles $\theta=\theta_{C} \pm 10^{\circ}$. In the top panel of Fig. 10 we also show parameterizations of the frequency spectrum of $100 \mathrm{PeV}$ protoninduced showers as obtained in [33]. Those parameterizations are based on 1-dimensional simulations and the lateral distribution was only acccounted for in a very approximate way, which explains the large discrepancies with the results of this work at the Cherenkov angle. However, the fits are in fairly good agreement for angles away from the Cherenkov angle since the features of the spectrum at those angles are determined by the longitudinal development of the shower which is correctly accounted for in [33] as well as in this work.

\subsection{Contribution of charged pions, muons and protons to excess track lengths}

In a hadronic shower, there are more particles besides electrons that can be expected to contribute to the excess charge. In fact, it has been experimentally determined [57] in atmospheric showers that cascades develop an excess of positive muons over negative muons, stemming from an excess of positively charged pions and kaons [58]. As a result, we expect pions and muons to contribute with an excess of positive track lengths to the Cherenkov radio emission.

In Fig. 11 we show the average longitudinal profile of electrons, positrons, protons, anti-protons, $\pi^{+}, \pi^{-}, \mu^{+}, \mu^{-}, K^{+}$and $K^{-}$as obtained in ZHAIRES simulations of 20 proton-induced showers of $E_{0}=1 \mathrm{PeV}$ in ice. It becomes apparent that the main contributions to the total projected tracklength, which is approximately propor- 

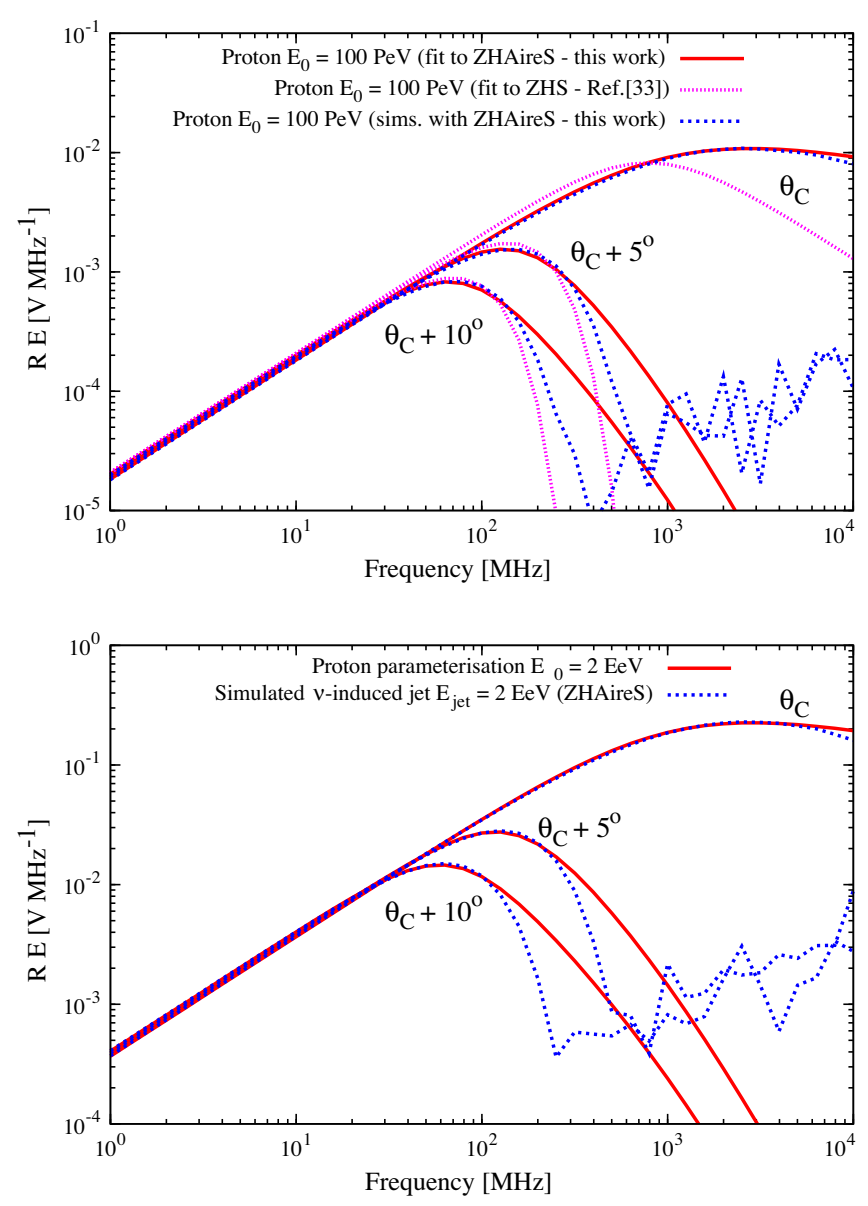

Fig. 10. Top panel: Frequency spectrum of the Cherenkov radiation obtained in ZHAIRES simulations of a proton shower with primary energy $E_{0}=100 \mathrm{PeV}$ (dashed blue line) at the Cherenkov angle and $5^{\circ}$ and $10^{\circ}$ away from it. The numerical results are compared to the parameterisations for proton-induced showers given in Appendix A (solid red lines) and in [33] (dotted magenta line). Bottom panel: Frequency spectrum obtained in ZHAIRES simulations of a neutrino-induced shower in a neutral current interaction (or a charged current interaction of a muon neutrino) in which the secondaries from the fragmentation of the nucleon carry an energy $E_{\text {jet }}=2 \mathrm{EeV}$ (dashed lines). The frequency spectrum is compared to the parameteresation of a proton shower with energy $E_{0}=2 \mathrm{EeV}$ (solid line) at different observation angles. See Section 4 for further details. (For interpretation of the references to colour in this figure legend, the reader is referred to the web version of this article.)

tional to the area under the longitudinal profile, come from charged pions, protons and muons. We neglect the contribution from charged kaons, which amounts to $\sim 1 \%$ of that due to pions. Protons contribute most to the excess positive tracklength because almost no anti-protons are produced in the shower to compensate for them, as can be seen in Fig. 11.

In order to quantify the influence of charged pions, muons and protons on the track lengths, we computed both, the total and projected track lengths of those particles in ZHAIRES simulations. As expected, we found that there is an excess of track lengths due to positively charged pions, muons and protons. For example, in a $10 \mathrm{PeV}$ proton shower:

$R_{T}=\frac{\left(T_{\pi^{+}}-T_{\pi^{-}}\right)+\left(T_{\mu^{+}}-T_{\mu^{-}}\right)+\left(T_{p}-T_{\bar{p}}\right)}{\left(T_{e^{-}}-T_{e^{+}}\right)} \sim 0.015$

where $T$ denotes the projected track length due to different particles. This means that the excess of positively charged pions, muons and protons diminishes the excess projected track length of the shower by $\sim 1.5 \%$ because the excess is of opposite sign, compared to the excess of electrons. This contribution decreases in magnitude

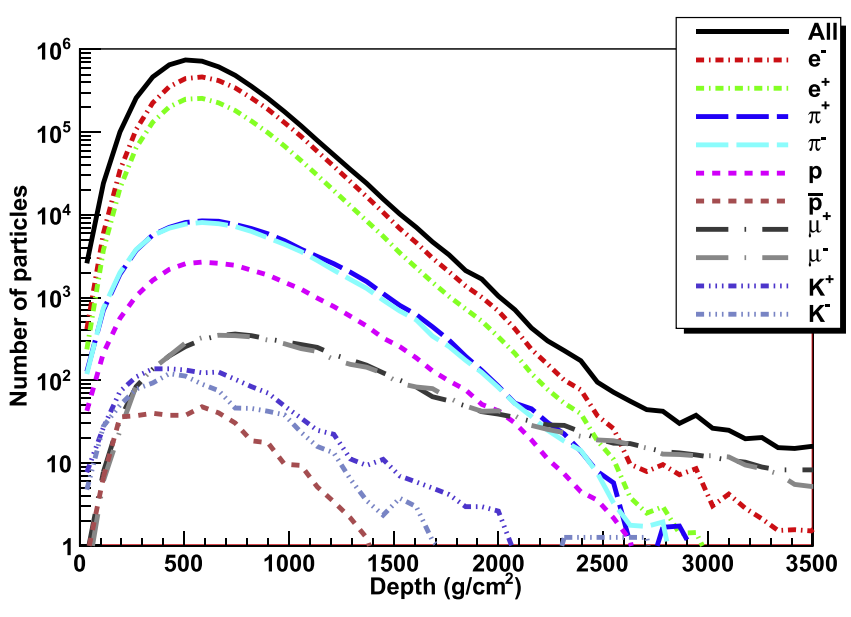

Fig. 11. Longitudinal development of of electrons, positrons, protons, anti-protons, $\pi^{+}, \pi^{-}, \mu^{+}, \mu^{-}, K^{+}$and $K^{-}$as obtained in ZHAIRES simulations of proton-induced showers in ice at $1 \mathrm{PeV}$.

with shower energy in the same fashion as the EM energy ratio increases (see Fig. 7 and Table 2). Also, for a fixed shower energy, there is a dependence on the hadronic interaction model used in the simulations, as will be discussed in the next section.

In the top half of Table 2 we show the total and projected track length divided by the primary energy $E_{0}$ due to different particles in proton-induced showers of $E_{0}=1 \mathrm{TeV}, 1 \mathrm{PeV}$ and $1 \mathrm{EeV}$. Note that the $e^{ \pm}$track lengths normalized to $E_{0}$ increase with energy, since the energy feeding the electromagnetic component of the shower also increases with the energy of the primary proton. This is illustrated by the EM energy fractions shown in the bottom of Table 2. As discussed before, this increase in the EM energy ratio is what causes the proton-induced shower projected excess $e^{ \pm}$ tracks to increase with energy, as can be seen in Fig. 7. Furthermore, both the total $e^{ \pm} \operatorname{track} / E_{0}$ and the projected $e^{ \pm} \operatorname{track} / E_{0}$ are proportional to the EM energy fraction of the shower. The ratio between the total $e^{ \pm}$track length normalized to $E_{0}$, and the EM energy fraction of the shower is constant, i.e. $\left(\sum T_{e^{ \pm}} / E_{0}\right) / E M^{\text {fraction }} \simeq 57 \mathrm{~m} /$ $\mathrm{TeV}$. The equivalent ratio for the projected $e^{ \pm}$tracks varies only slightly between 41 and $45 \mathrm{~m} / \mathrm{TeV}$ for all the energy range shown in Table 2.

The very small contribution of pions, muons and protons to the field can be seen in Fig. 12, where we show the Fourier-spectrum of the electric field in proton showers of $10 \mathrm{PeV}$ with and without accounting for the charged pions, muons and protons tracks. Although the actual field emitted by the hadrons in the shower is very small, compared to the field emitted by electrons and positrons, the hadronic component carries a significant fraction of the shower energy, especially at lower energies. The importance of this energy balance of the shower can be illustrated by the effects of the EM energy fraction on the radio emission, as discussed before. Given our results, following the actual tracks of hadrons in the shower turns out not to be essential for the field calculation, but accounting for the hadronic energy of the shower is.

\subsection{Hadronic interaction model dependence}

Modeling of hadronic showers above PeV energies relies on the extrapolation of hadronic interactions to energies and regions in the parameter space of the collisions which have not been probed in terrestrial accelerators [59]. We have studied the influence of the hadronic model on the coherent Cherenkov radiation frequency spectrum. For that purpose we have simulated a set of proton-induced showers with the QGSJET01 model [60], and compared the spectrum with that obtained using the SIBYLL 2.1 model [61], which 


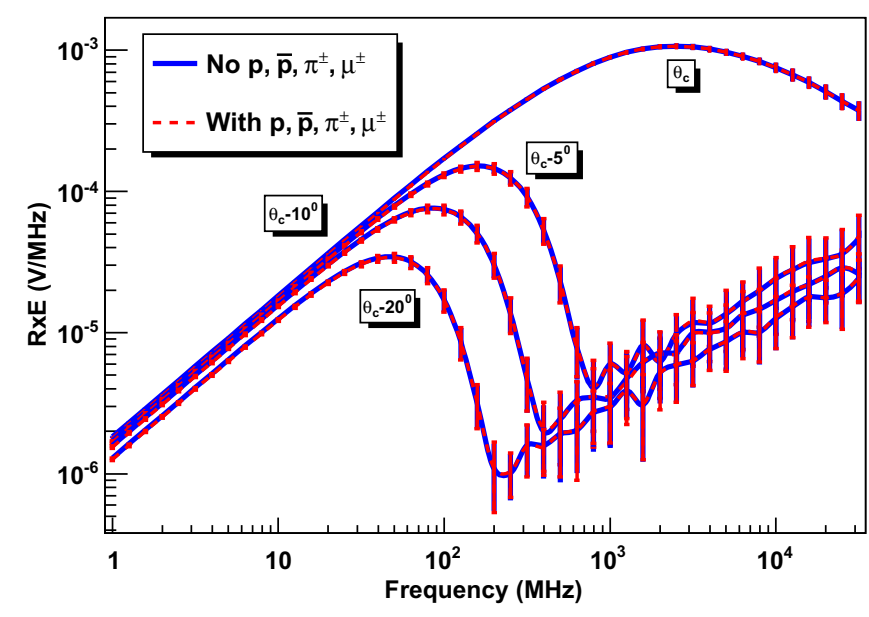

Fig. 12. Average frequency spectrum of the Cherenkov radiation obtained from ZHAIRES simulations of $10 \mathrm{PeV}$ proton-induced showers accounting (red dashed lines) and not accounting (blue solid line) for pions, muons and protons in the calculation of the electric field. The spectrum is shown at four observation angles with respect to shower axis. The RMS of the 20 simulated showers is also shown. (For interpretation of the references to colour in this figure legend, the reader is referred to the web version of this article.)

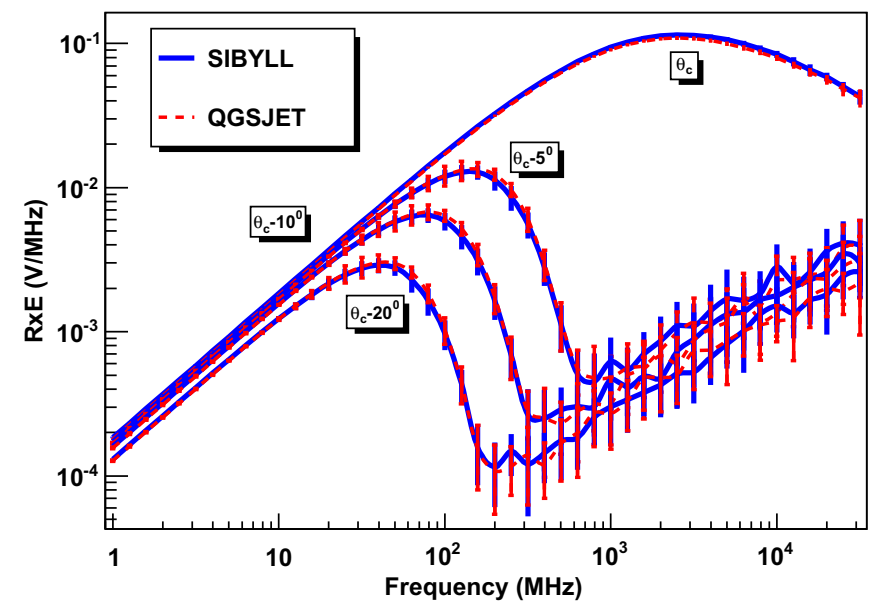

Fig. 13. Average frequency spectrum of the Cherenkov radiation obtained from ZHAIRES simulations of $1 \mathrm{EeV}$ proton-induced showers using QGSJET01 (red dashed lines) and SIBYLL 2.1 (blue solid line). The spectrum is shown at four observation angles with respect to shower axis. The RMS of the 20 simulated showers is also shown. (For interpretation of the references to colour in this figure legend, the reader is referred to the web version of this article.)

we have been using by default in all other simulations so far ${ }^{6}$. This comparison is shown in Fig. 13. The differences between the normalization of the spectra in the coherent region obtained with SIBYLL 2.1 and QGSJET01 are small, with the QGSJET01 spectrum smaller than that predicted with SIBYLL 2.1 by up to $\sim 4 \%$ around the cutoff frequency at the Cherenkov angle.

It is well known that QGSJET01 produces more muons and pions per interaction than SIBYLL 2.1 [63] and also protons. For instance, we have obtained the longitudinal profile of the number of pions in proton-induced showers, and observed that the number of pions at the maximum is $\sim 24 \%$ larger in QGSJET01 than in SIBYLL 2.1 [63]. Also more protons, pions, and muons per interaction means less electrons and positrons in the shower, decreasing the negative excess track (denominator of Eq. (3) ). As a consequence, we expect a difference in the ratio $R_{T}$ predicted by both models. While in SIBYLL

${ }^{6}$ We use the SIBYLL 2.1 and QGSJET01 models since recent data favors them over
other models [62].
$2.1 R_{T} \sim 0.012$ at $1 \mathrm{EeV}, \mathrm{QGSJET0} 1$ predicts a larger ratio $R_{T} \sim 0.014$, the increase being dominated by the proton projected track-length. Also as a consequence, the EM energy ratio is larger (93\%) in SIBYLL when compared to QGSJET (91\%) at $1 \mathrm{EeV}$. This is consistent with the fact that the electric field in the fully coherent region is slightly smaller when using QGSJET01 than when SIBYLL 2.1 is adopted. We remark here that the smallness of $R_{T}$ reflects that the tracks of protons, pions and muons are mostly irrelevant to the field calculation, and this conclusion holds regardless of the details of the high-energy hadronic interaction model. In fact, we have also studied the effect of the low energy hadronic models on the radio emission, and our conclusions remain the same.

There are also differences in the frequency at which the electric field intensity is maximum at each observation angle away from the Cherenkov angle. These stem from the fact that the longitudinal profile of the excess negative charge predicted by SIBYLL 2.1 is longer than in QGSJET01, leading to higher frequencies at the maximum when using QGSJET01. Also, the lateral profile is slightly flatter in QGSJET, leading to a slightly smaller cut-off frequency at the Cherenkov angle. However, these differences are small.

\section{Summary and conclusions}

We have presented ZHAIRES, a Monte Carlo code that merges the high energy hadronic interaction and tracking capabilities of AIRES[44], and the dense media propagation capabilities of TIERRAS [45] with the precise low energy $e^{ \pm}$tracking and radiation calculation capabilities of ZHS[9,25]. This combination allows a precise full simulation of radio emission of electromagnetic, and for the first time also hadronic showers up to EeV energies. ZHAIRES has been used in conjuction with the TIERRAS package [45] to simulate showers in dense media, obtaining the radiation emitted due to the Askar'yan effect.

We have compared ZHAIRES results for electromagnetic showers in ice against ZHS up to $10 \mathrm{EeV}$, obtaining a good agreement between them. In the case of hadronic showers, we have compared the results from ZHAIRES and GEANT4 up to $90 \mathrm{TeV}$ [28], the highest energy for full simulations of hadronic showers with GEANT in the literature, also with very good quantitative agreement. Unlike GEANT4, ZHAIRES is capable of simulating the emission from hadronic showers up to EeV energies.

By comparing the results of thinned ZHAIRES simulations with un-thinned ones up to $1 \mathrm{PeV}$, we obtained thinning parameters which give a good compromise between accuracy and CPU time. We then tested the applicability of these parameters at higher energies, by comparing our results against ZHS, which has its own, well established [32], thinning algorithms and parameters.

We confirmed the expectation that the EM energy ratio of hadronic showers keeps approaching that of EM showers at the highest energies, increasing the magnitude of the electric field (normalized by $E_{0}$ ) as energy grows. We also showed that, in the case of UHE purely EM showers, the EM energy ratio deviates from a linear dependence on energy, due to photohadronic interactions.

By comparing EM and hadronic showers up to $10 \mathrm{EeV}$ we have found that, at the Cherenkov angle, the cut-off frequency tends to be smaller in hadronic showers and increases slowly as the energy rises because the lateral distribution becomes narrower. For angles away from the Cherenkov angle, as expected, the cut-off frequency decreases with energy, due to the logarithmic growth of the longitudinal profile. In contrast, away from the Cherenkov angle, the cut-off frequency for EM showers decreases rapidly above PeV energies due to the LPM effect, which is much more pronounced in EM showers than in hadronic ones. We also found that the fluctuations in hadronic showers are almost independent of energy, while in EM showers they grow rapidly, as the LPM effect becomes important. 
We have also analyzed the influence of charged pions, muons and protons on the radio emission of showers. We found that most of their contribution is due to the excess of protons, with pions being the next contribution in importance, that induce a decrease of the (total) negative excess track length of the shower of $\sim 1-2 \%$ above $1 \mathrm{PeV}$. Although the actual field emitted by the hadrons in the shower is very small, the hadronic component carries a significant fraction of the shower energy. The importance of this energy balance is illustrated by the effects of the EM energy fraction on the radio emission. Given our results, following the actual tracks of hadrons in the shower turns out not to be essential for the field calculation, but accounting for the hadronic energy of the shower is.

We have compared the results obtained using SIBYLL 2.1 [61] and QGSJET01 [60], and found that there is a small dependence of the normalization of the emitted electric field on the hadronic model, up to $4 \%$ lower in QGSJET01 at the Cherenkov angle. This is due to the higher number of protons, pions and muons when QGSJET01 is used, which increases the positive projected excess track-length of protons, pions and muons, with protons dominating the difference. Also more protons, pions and muons means less electrons and positrons in the shower, decreasing the negative excess track. The net effect is a lower negative projected excess track as a whole, diminishing the field. At angles away from the Cherenkov angle, the longitudinal excess profile becomes important, and the shorter showers obtained using QGSJET lead to the maximum of the emission at higher frequencies, when compared to SIBYLL showers.

Finally, we have used our simulations of the emission due to the Askar'yan effect in hadronic showers to obtain new parameterizations of the radio pulse frequency spectrum which are described in Appendix A. We note that our results for proton showers can also be applied to approximately model the Cherenkov emission from hadronic showers induced in high-energy neutrino interactions. This is shown in the bottom panel of Fig. 10 where we have plotted the frequency spectrum obtained in ZHAIRES simulations of a neutrino-induced shower in a neutral current interaction (or a charged current interaction of a muon neutrino) in which the secondaries from the fragmentation of the nucleon carry $2 \mathrm{EeV}$ of energy in total. The products of the neutrino-nucleon interaction, mainly pions, were obtained with the HERWIG interaction Monte Carlo code [64]. The frequency spectrum obtained in ZHAIRES simulations is compared to the parameterization of a proton shower with energy $E_{0}=2 \mathrm{EeV}$ as given in Appendix A.

\section{Acknowledgments}

J.A-M, W.R.C. and E.Z. thank Xunta de Galicia (INCITE09 206336 PR) and Consellería de Educación (Grupos de Referencia Competitivos - Consolider Xunta de Galicia 2006/51); Ministerio de Ciencia e Innovación (FPA 2007-65114, FPA 2008-01177 and Consolider CPAN) and Feder Funds, Spain. We thank CESGA (Centro de SuperComputación de Galicia) for computing resources and assistance. M.T. thanks Fundación Universidad Nacional de Cuyo and CONICET (Argentina) for financial support. We thank J. Bray, R. Crocker, C.W. James, R.J. Protheroe, A. Romero-Wolf and R.A. Vázquez for many helpful discussions. Special thanks to Gonzalo Rodríguez-Fernández for help with GEANT4 simulations.

\section{Appendix A}

In this Appendix we give parameterizations of the magnitude of the Cherenkov electric field in proton-induced showers in ice for practical applications, as obtained in simulations with ZHAIRES.
This is the first time parameterizations of the electric field in hadronic showers are presented. The parameterizations given here complement those presented in [32] for purely electromagnetic showers. However in [32] photoproduction interactions were not taken into account, and for this reason the parameterizations presented there for electromagnetic showers should be less accurate for $E>10^{19} \mathrm{eV}$. This important subject will be further studied in a future work.

The physical basis for our parameterization of the radiated spectrum is the "box model" of shower development $[25,41,7,32]$. In this model, the distribution of particle tracks has a characteristic length $L$, proportional to the radiation length $X_{0}$, and a width $R$, proportional to the Molière radius $R_{M}$. The model motivated a functional form for the radiated spectrum $\vec{E}(v)$ in electromagnetic showers, given by [32]:

$r\left|\vec{E}\left(E_{0}, \theta, v\right)\right|=A\left(E_{0}, \theta, v\right) \times d_{L}\left(E_{0}, \theta, v\right) \times d_{R}\left(E_{0}, \theta, v\right)$

where $A$ is a coherent amplitude that increases linearly with frequency, which is multiplied by lateral and longitudinal decoherence factors $d_{R}$ and $d_{L}$, defined in such a way so that $d_{R, L} \leqslant 1$ and $d_{R, L} \rightarrow 1$ as $v \rightarrow 0$. Also, as $\theta \rightarrow \theta_{C}$, where $\theta_{C}$ is the Cherenkov angle, $d_{L} \rightarrow 1$, since interference over the width of the shower dominates. In general, $A, d_{L}$ and $d_{R}$ depend on the shower energy $E_{0}$, frequency $v$, and observation angle $\theta$ with respect to the shower axis. $r$ is the (farfield) observation distance.

In this paper we adopt this same functional form for the radiated spectra in proton-induced showers. For the decoherence factors we use the same functional forms as in [7], namely:

$d_{R[L]}=\frac{1}{1+\left(v / v_{R[L]}\right)^{\bar{\alpha}[\beta]}}$.

where $v_{R}$ and $v_{L}$ are characteristic frequencies at which lateral and longitudinal decoherence become important, and $\bar{\alpha}$ and $\bar{\beta}$ give the strength of the decoherence. The frequencies $v_{L}$ and $v_{R}$ are inversely proportional to $L$ and $R$, respectively. The amplitude $A$ of the fully coherent (low-frequency) component is proportional to the total excess track length $T$, which in hadronic showers is known to deviate from a linear behavior with shower energy (see Fig. 7). The length $L$, width $R$, and excess track length $T$ of the shower can be related to properties of the interaction medium $[25,41,31,7]$ :

$$
\begin{aligned}
& v_{L}(\theta) \approx \frac{c}{L} \frac{1}{|1-n \cos \theta|}=\frac{\rho}{\bar{k}_{L} X_{0}} \frac{c}{|1-n \cos \theta|} \\
& v_{R}\left(\theta=\theta_{C}\right) \approx \frac{c / n}{R}=\frac{\rho}{\bar{k}_{R} R_{M}} \frac{c}{\sqrt{n^{2}-1}} \\
& A\left(E_{0}, \theta, v\right)=\bar{k}_{E} v T \sin \theta \approx \bar{k}_{E} \frac{E_{0}}{E_{C}} \frac{X_{0}}{\rho} v_{\mathrm{MHz}} \sin \theta
\end{aligned}
$$

where $v_{\mathrm{MHz}}$ is the frequency in $\mathrm{MHz}, \bar{k}_{E}$ has units of $\mathrm{V} \mathrm{cm}^{-1} \mathrm{MHz}^{-2}$ and $c=3 \cdot 10^{10} \mathrm{~cm} \mathrm{~s}^{-1}$ is the speed of light. $X_{0}=36.08 \mathrm{~g} \mathrm{~cm}^{-2}$, $R_{M}=10.57 \mathrm{~g} \mathrm{~cm}^{-2}, n=1.78, \rho=0.924 \mathrm{~g} \mathrm{~cm}^{-3}$ and $E_{C}=73.1 \mathrm{MeV}$ are respectively the radiation length, Molière radius, refractive index, density, and critical energy of ice. Throughout, we use the approximation $v_{R}(\theta)=v_{R}\left(\theta_{C}\right)$, since lateral decoherence is only important near the Cherenkov angle.

The quantity $\bar{k}_{E}$ is also energy dependent, since the track length due to the excess negative charge deviates from linearity in hadronic showers (see Fig. 7). To fit $\bar{k}_{E}\left(E_{0}\right)$ we use:

$\bar{k}_{E}=k_{E, 0} \tanh \left(\frac{\log _{10} E_{0}-\log _{10} E_{E}}{k_{E, 1}}\right)$

where $k_{E, 0}, k_{E, 1}$ and $E_{E}$ are constants given in Table 3 .

Since $L$ is dependent upon the shower energy $E_{0}, \bar{k}_{L}$ is in fact also energy-dependent, although with a much weaker dependence than in electromagnetic showers, because hadronic showers are not so 
Table 3

Fitted proton-induced shower parameters in ice, as defined by Eq. (9).

\begin{tabular}{lll}
\hline$k_{E, 0}\left[\mathrm{~V} \mathrm{~cm}^{-1} \mathrm{MHz}^{-2}\right]$ & $k_{E, 1}$ & $\log _{10}\left(E_{E} / \mathrm{eV}\right)$ \\
\hline $4.13 \times 10^{-16}$ & 2.54 & 10.60 \\
\hline
\end{tabular}

Table 4

Fitted proton-induced shower parameters in ice, as defined by Eq. (10).

\begin{tabular}{lll}
\hline$k_{L, 0}$ & $\gamma$ & $\log _{10}\left(E_{L} / \mathrm{eV}\right)$ \\
\hline 31.25 & $3.01 \times 10^{-2}$ & 15.00
\end{tabular}

Table 5

Fitted proton-induced shower parameters in ice, as defined by Eq. (11).

\begin{tabular}{lll}
\hline$k_{R, 0}$ & $k_{R, 1}$ & $\log _{10}\left(E_{R} / \mathrm{eV}\right)$ \\
\hline 2.73 & 1.72 & 12.92 \\
\hline
\end{tabular}

Table 6

Fitted proton-induced shower parameters in ice, as defined by Eq. (5).

\begin{tabular}{ll}
\hline $\bar{\alpha}$ & $\bar{\beta}$ \\
\hline 1.27 & 2.57 \\
\hline
\end{tabular}

strongly affected by the LPM effect. For this reason we expect $\bar{k}_{L}$ to be well fit in the whole energy range by the relation:

$\bar{k}_{L}=k_{L, 0}\left(\frac{E}{E_{L}}\right)^{\gamma}$

where $k_{L, 0}, E_{L}$ and $\gamma$ are constants given in Table 4 .

We also found a weak dependence of $\bar{k}_{R}$ with energy, which we further parameterize as:

$\bar{k}_{R}=k_{R, 0}+\tanh \left(\frac{\log _{10} E_{R}-\log _{10} E_{0}}{k_{R, 1}}\right)$

where $k_{R, 0}, k_{R, 1}$ and $E_{R}$ are constants given in Table 5 .

Finally, $\bar{\alpha}$ and $\bar{\beta}$ are found to be practically independent of shower energy, and they are given in Table 6 .

Our fitting procedure is the same as in [32]. We reproduce it here for self-consistency of the paper. For each shower of energy $E_{0}$, we start by obtaining $A(\theta, v)$ and $d_{R}$ from a fit to the spectrum at the Cherenkov angle $\theta_{C}$ where $d_{L}=1$. We then obtain $\bar{k}_{E}$ by substituting $A(\theta, v)$ in Eq. (8). Also from the fitted $d_{R}$ in Eq. (5), we obtain parameters $v_{R}$ and $\bar{\alpha}$, and relate $v_{R}$ to $\bar{k}_{R}$ via Eq. (7). Fixing $A$ and $d_{R}$ to their fitted values, we then allow $d_{L}$ to vary on fits of Eq. (4) to the simulated field at various angles away from the Cherenkov angle, obtaining $d_{L}(\theta, v)$ for each angle. From these we can obtain $v_{L}(\theta)$ and $\bar{\beta}$ from Eq. (5), and $\bar{k}_{L}$ from Eq. (6), respectively. We repeat this process many times, varying the energy $E_{0}$ in multiples of 10 over the energy range from $1 \mathrm{TeV}$ to $10 \mathrm{EeV}$. By using the values of $\bar{k}_{E}\left(E_{0}\right), \bar{k}_{L}\left(E_{0}\right)$ and $\bar{k}_{R}\left(E_{0}\right)$ from fits at these different energies, we obtain the parameters of Eqs. (9)-(11), that define the energy dependence of $\bar{k}_{E}, \bar{k}_{L}$ and $\bar{k}_{R}$, and are given in Tables $3-5$, respectively. The fitted parameters $\bar{\alpha}$ and $\bar{\beta}$, practically energy independent, are given in Table 6 .

While all the fitted parameters vary on a shower-to-shower basis, variations tended to be small, so that approximating $k_{E} \approx \bar{k}_{E}, \beta \approx \bar{\beta}$, etc., is appropriate for individual showers. This is in contrast to previous parameterizations of electromagnetic showers, in which $k_{L}$ varied strongly from the mean fitted values $\bar{k}_{L}$, due to variations in the longitudinal spread of the showers caused by the LPM effect. This is not the case in hadronic showers and we take $k_{L} \approx \bar{k}_{L}$.
Finally, the fits have an accuracy of $\sim 1 \%$ for frequencies up to the frequency $v_{\max }$, at which the spectrum is maximum for each observation angle. For frequencies above $v_{\max }$, the accuracy worsens gradually and reaches $\sim 5 \%$ at $v=2 v_{\max }$, for observation at the Cherenkov angle $\theta=\theta_{C}$, and $\sim 15 \%$ at angles $\theta=\theta_{C} \pm 10^{\circ}$.

\section{References}

[1] J.K. Becker, Phys. Rep. 458 (2008) 173. and Refs. therein.

[2] G.A. Askar'yan, Soviet Phys. JETP 14 (2) (1962) 441-443; G.A. Askar'yan, Soviet Phys. JETP 48 (1965) 988-990.

[3] D. Saltzberg et al., Phys. Rev. Lett. 86 (2001) 2802.

[4] P. Miocinovic et al., Phys. Rev. D 74 (2006) 043002.

[5] P.W. Gorham et al., Phys. Rev. D 72 (2005) 023002.

[6] P.W. Gorham et al., Phys. Rev. Lett. 99 (2007) 171101.

[7] J. Alvarez-Muñiz, E. Marqués, R.A. Vázquez, E. Zas, Phys. Rev. D 74 (2006) 023007.

[8] R.D. Dagkesamansky, I.M. Zheleznykh, in: M. Nagano, F. Takahara (Eds.), Proceedings of the ICRR international symposium: astrophysical aspects of the most energetic cosmic rays (Kofu, Japan, November 1990), World Scientific (1991) p. 373.

[9] F. Halzen, E. Zas, T. Stanev, Phys. Lett. B 257 (1991) 432.

[10] G.M. Frichter, J.P. Ralston, D.W. McKay, Phys. Rev. D 53 (1996) 1684

[11] S. Barwick et al., Phys. Rev. Lett. 96 (2006) 171101.

12] P.W. Gorham et al., Astropart. Phys. 32 (2009) 10.

[13] P.W. Gorham et al., Phys. Rev. Lett. 103 (2009) 051103.

[14] I. Kravchenkov et al., Astropart. Phys. 19 (2003) 15.

[15] I. Kravchenko et al., Phys. Rev. D 73 (2006) 082002.

[16] The ARA Collaboration: ARA proposal. Instrument Development of the Askaryan Radio Array, a Large-scale Radio Cherenkov Detector at the South Pole, available at: http://grid.ntu.edu.tw/html/projects/pro101/pro101.pdf.

[17] S.W. Barwick et al., ARIANNA Collaboration, J. Phys. Conf. Ser. 60 (2007) 276286.

[18] T. Hankins et al., Mon. Not. Royal Astron. Soc. 283 (1996) 1027.

[19] C.W. James et al., Mon. Not. Royal Astron. Soc. 379 (2007) 1037.

[20] P.W. Gorham et al., Phys. Rev. Lett. 93 (2004) 041101.

[21] A.R. Beresnyak et al., Astron. Reports 49 (2) (2005) 127.

[22] O. Scholten et al., Astropart. Phys. 26 (2006) 219.

[23] C.W. James et al., Phys. Rev. D 81 (2010) 042003.

[24] T.R. Jaeger, R. L Mutel, K.G. Gayley, Astropart. Phys. 34 (2010) 293.

[25] E. Zas, F. Halzen, T. Stanev, Phys. Rev. D 45 (1992) 362.

[26] S. Razzaque et al., Phys. Rev. D 69 (2004) 047101.

[27] J. Alvarez-Muñiz, E. Marqués, R.A. Vázquez, E. Zas, Phys. Rev. D 67 (2003) 101303.

[28] S. Hussain, D.W. McKay, Phys. Rev. D 70 (2004) 103003.

[29] J. Alvarez-Muñiz et al., Phys. Rev. D 66 (2002) 033011.

[30] A.M. Hillas, Nucl. Phys. B (Proc. Suppl.) 52B (1997) 29.

[31] J. Alvarez-Muñiz, E. Zas, Phys. Lett. B 411 (1997) 218.

[32] J. Alvarez-Muñiz, C.W. James, R.J. Protheroe, E. Zas, Astropart. Phys. 32 (2009) 100.

[33] J. Alvarez-Muñiz, E. Zas, Phys. Lett. B 434 (1998) 396.

[34] S. Bevan et al., Astropart. Phys. 28 (2007) 366. see also S. Bevan et al. astro-ph/ $0704.1025 \mathrm{v} 1$.

[35] J. Alvarez-Muñiz, R.A. Vázquez, E. Zas, Phys. Rev. D 61 (1999) 023001.

[36] L. Landau, I. Pomeranchuk, Dokl. Akad. Nauk SSSR 92 (1953) 535; L. Landau, I. Pomeranchuk, Dokl. Akad. Nauk SSSR 92 (1935) 735; A.B. Migdal, Phys. Rev. 103 (1956) 1811;

A.B. Migdal, Zh. Eksp. Teor. Fiz. 32 (1957) 633 A.B. Migdal, Sov. Phys. JETP 5 (1957) 527.

[37] T. Stanev et al., Phys. Rev. D 25 (1982) 1291.

[38] J.P. Ralston, D.W. McKay, in: James Matthews (Ed.), Proceedings of high energy gamma-ray astronomy conference (Ann Arbor, Mi 1990), (AIP Conf. Proc. 220), p. 295.

[39] J. Alvarez-Muñiz, R.A. Vázquez, E. Zas, Phys. Rev. D 62 (2000) 063001.

[40] Klein, Gerhardt, Phys. Rev. D 82 (2010) 074017.

[41] R.V. Buniy, J.P. Ralston, Phys. Rev. D 65 (2002) 016003

[42] J. Alvarez-Muñiz, A. Romero-Wolf, E. Zas, Phys. Rev. D 81 (2010) 123009

[43] J. Alvarez-Muñiz et al., in: Proceedings of the 31st International Cosmic Ray Conference, Lodz, Poland (2009), p. 581.

[44] S. Sciutto, AIRES manual, http://www.fisica.unlp.edu.ar/auger/aires/.

[45] M. Tueros, S. Sciutto, Comp. Phys. Comm. 181 (2010) 380.

[46] See GEANT4 Manual available in <http://geant4.web.cern.ch/geant4/>

[47] S. Razzaque et al., Phys. Rev. D 65 (2002) 103002;

[48] H.R. Allan, Progress in Elementary Particles and Cosmic Ray Physics 10, 171 (1971), North Holland Publ. Co.), and Refs. therein.

[49] A. Haungs, Nucl. Instr. Meth. Phys. Res. 604 (S236) (2009), and Refs. therein; T. Huege, M. Ludwig, Astropart. Phys. 34 (2011) 438.

[50] J. Alvarez-Muñiz, W.R. Carvalho, E. Zas, A. Romero-Wolf, M. Tueros, in: Proceedings of the ARENA 2010 meeting, Nantes, France, Nucl. Instr. Meth. Phys. Res. A, in press.

[51] J. Alvarez-Muñiz, W.R. Carvalho, E. Zas, arXiv:1107.1189v1 [astro-ph.HE], (2011).

[52] J. Alvarez-Muñiz et al. in preparation.

[53] A.N. Cillis et al., Phys. Rev. D 59 (1999) 113012. 
[55] L. Gerhardt, S.R. Klein, Phys. Rev. D 82 (2010) 074017.

[56] J.P. Ralston et al., (2002), arXiv:astro-ph/0209455.;

S.R. Klein, Radiat. Phys. Chem. 75 (2006) 696.

[57] P. Adamson et al., Phys. Rev. D 76 (2007) 052003.

[58] T.K. Gaisser, Cosmic Rays and Particle Physics, Cambridge Univ. Press, 1992.

[59] C.A. García Canal et al., Phys. Rev. D 79 (2009) 054006. and Refs. therein.

[60] N.N. Kalmykov, S.S. Ostapchenko, A.I. Pavlov, Nucl. Phys. Proc. Suppl. 52B (1997) 17.
[61] R.S. Fletcher, T.K. Gaisser, P. Lipari, T. Stanev, Phys. Rev. D63 (2001) 054030

[62] J.R. Hörandel et al., in: Proceedings 31st international cosmic ray conference, Lodz, Poland (2009), p. 227.

[63] S.S. Ostapchenko, Czech. J. Phys. 56 (2006) A149. hep-ph/0601230.

[64] G. Corcella et al., Herwig 6.5, JHEP 0101 (2001). 